Draft version March 11, 2021

Preprint typeset using $\mathrm{L}^{A} \mathrm{~T}_{\mathrm{E}} \mathrm{X}$ style emulateapj v. 08/22/09

\title{
DIRECT MEASUREMENT OF QUASAR OUTFLOW WIND ACCELERATION ${ }^{1,2,3}$
}

\author{
Toru Misawa ${ }^{4}$, Michael Eracleous ${ }^{5,6}$, Jane C. Charlton $^{5}$, and Nobunari Kashikawa ${ }^{7}$ \\ ${ }^{4}$ School of General Education, Shinshu University, 3-1-1 Asahi, Matsumoto, Nagano 390-8621, Japan; misawatr@shinshu-u.ac.jp \\ ${ }^{5}$ Department of Astronomy \& Astrophysics, Pennsylvania State University, 525 Davey Lab, University Park, PA 16802 \\ ${ }^{6}$ Institute for Gravitation and the Cosmos, Pennsylvania State University, 525 Davey Lab, University Park, PA 16802 \\ ${ }^{7}$ Department of Astronomy, School of Science, The University of Tokyo, 7-3-1 Hongo, Bunkyo-ku, Tokyo 113-0033, Japan
}

Draft version March 11, 2021

\section{ABSTRACT}

We search for velocity changes (i.e., acceleration/deceleration) of narrow absorption lines (NALs) that are intrinsic to the quasars, using spectra of 6 bright quasars that have been observed more than once with $8-10 \mathrm{~m}$ class telescopes. While variations in line strength and profile are frequently reported (especially in broader absorption lines), definitive evidence for velocity shifts has not been found with only a few exceptions. Direct velocity shift measurements are valuable constraints on the acceleration mechanisms. In this study, we determine velocity shifts by comparing the absorption profiles of NALs at two epochs separated by more than 10 years in the observed frame, using the cross-correlation function method and we estimate the uncertainties using Monte Carlo simulations. We do not detect any significant shifts but we obtain $3 \sigma$ upper limits on the acceleration of intrinsic NALs (compared to intervening NALs in same quasars) of $\sim 0.7 \mathrm{~km} \mathrm{~s}^{-1} \mathrm{yr}^{-1}\left(\sim 0.002 \mathrm{~cm} \mathrm{~s}^{-2}\right)$. We discuss possible scenarios for non-detection of NAL acceleration/deceleration and examine resulting constraints on the physical conditions in accretion disk wind.

Subject headings: quasars: absorption lines - quasars: individual (HE0130-4021, Q0450-1310, HE0940-1050, HE1009+2956, Q1107+4847, and HS1700+6416)

\section{INTRODUCTION}

The signatures of outflowing winds from accretion disks are observed in the spectra of $\sim 50 \%$ of quasars and active galactic nuclei (hereafter AGNs; Hamann et al. 2012). The winds play an important role in providing energy and momentum feedback to the interstellar medium (ISM) of their host galaxies and the intergalactic medium (IGM), and by transporting heavy elements out of the host galaxies (e.g., Springel et al. 2005). The blueshifted near-UV absorption lines (e.g., the C IV, $\mathrm{Si}$ IV, and N v doublets) that are the observational signature of outflowing winds, are usually classified into three categories according to their widths: broad absorption lines (BALs) with FWHM $\geq 2,000 \mathrm{~km} / \mathrm{s}$, narrow absorption lines (NALs) with FWHM $\leq 500 \mathrm{~km} / \mathrm{s}$, and an intermediate subclass (mini-BALs). The difference in line width may depend on the inclination angle of our line of sight relative to the axis of the wind (an orientation scenario; e.g., Weymann et al. 1991), although evolutionary scenarios have also been discussed (e.g., Lípari \& Terlevich 2006); see summary in Bruni et al. (2012) and references therein.

The physical conditions of quasar winds have been studied through variability of the absorption lines since

\footnotetext{
${ }^{1}$ Based on data collected at Subaru Telescope, which is operated by the National Astronomical Observatory of Japan.

2 Data presented herein were obtained at the W.M. Keck Observatory, which is operated as a scientific partnership among the California Institute of Technology, the University of California and the National Aeronautics and Space Administration. The Observatory was made possible by the generous financial support of the W.M. Keck Foundation.

3 Based on observations obtained at the European Southern Observatory at La Silla, Chile in programs 65.O-0474(A) and 079.B0469(A)
}

almost all BALs and mini-BALs vary over a few years in the quasar's rest-frame (e.g., Gibson et al. 2008; Misawa et al. 2014). Variations are usually seen in the strengths and profiles of (mini-)BAL troughs (e.g., Capellupo et al. 2012). The probability of observing variations increases with the time interval between observations, approaching unity for an interval of a few years (e.g., Capellupo et al. 2011). In extreme cases, BAL profiles appear or disappear completely (e.g., Vivek et al. 2012; Filiz Ak et al. 2012). Possible origins of such variations include (a) motion of the absorbing gas parcels across our line of sight (e.g., Hamann et al. 2008) and (b) changes in the ionization state of the absorber (e.g., Misawa et al. 2007b).

Velocity shifts of absorption lines from winds have not been studied except in a few cases for BALs and mini-BALs (e.g., Joshi et al. 2018; Grier et al. 2016; Joshi et al. 2014), despite the fact that acceleration is a key physical property of the gas in quasar winds. A direct detection of velocity shifts would provide valuable constraints on acceleration mechanisms. Three different processes have been proposed for accelerating winds: radiation pressure (absorption of line photons; e.g., Murrav et al. 1995), magnetocentrifugal forces (e.g., Everett 2005), and thermal pressure (e.g., Balsara \& Krolik 1993). Among them, radiation pressure must contribute significantly at least to the acceleration of X-ray absorbers with large ejection velocity (Ultra-Fast Outflows or UFOs), because their total kinetic energy, $\dot{E}_{k}=\frac{1}{2} \dot{M} v^{2}$ (where $\dot{M}$ is the mass ejection rate) is well correlated with the bolometric luminosity $\left(L_{b o l}\right)$ of the quasars (e.g., Tombesi et al. 2013). However, it is still not clear whether the radiative force is also the main source of acceleration for absorbers detected 
through $U V$ lines whose distances from the central engine may be much larger than those of absorbers detected via X-ray lines. Studies of velocity shifts using (mini-)BALs may be complicated by the following: (1) the absorption lines sometimes suffer from self-blending (i.e., the blue and red components of a doublet are blended with each other), and (2) a change in line profile could be mistaken as a velocity shift.

In contrast to previous studies that focused on BALs and mini-BALs, we monitor here the velocities of intrinsic NALs, for the first time. In the case of NALs, small velocity shifts can be detected very easily because of their small widths. We describe target selection in $\S 2$, the observations and data reduction in $\$ 3$, and the data analysis in $\S 4$. The results and discussion are presented in $\S 5$ and $\S 6$. We close by summarizing our results in $\S 7$. We use a cosmology with $H_{0}=70 \mathrm{~km} \mathrm{~s}^{-1} \mathrm{Mpc}^{-1}, \Omega_{m}=0.3$, and $\Omega_{\Lambda}=0.7$ throughout the paper.

\section{SAMPLE SELECTION}

We assembled a sample of six quasars based on two criteria: a) they have at least one $\mathrm{C}$ IV, Si IV, or N V NAL in their spectra that has already been confirmed to be physically associated with the quasar, and b) there are multiple high-resolution spectra $(R \sim 40,000)$ of each quasar that were already available or would soon be available through upcoming observing programs over a time interval of more than 10 years in the observed frame.

We selected the target quasars with "reliable" (class A) and "possible" (class B) intrinsic NALs from the catalog of Misawa et al. (2007a). The catalog was constructed after examining the NALs in 37 quasar spectra and selecting those that are intrinsic based on partial coverage analysis. Two of the quasars we selected, HE01304021 and HS1700+6416, had previously been observed more than once with $10 \mathrm{~m}$ class telescopes (i.e., the Keck and Subaru Telescopes, and the Very Large Telescope, hereafter VLT) with some time intervals that exceed 10 years in the observed frame (see details in Misawa et al. 2014). We also chose four additional quasars, Q0450 1310, HE0940-1050, HE1009+2956, and Q1107+4847, which we re-observed with the Subaru Telescope. These four quasars are known to have at least one intrinsic NAL and their oldest spectra were taken more than 10 years ago. They are accessible from the Subaru Telescope, and are bright enough $(V \leq 18.2)$ so that spectra with a signal-to-noise $(\mathrm{S} / \mathrm{N})$ ratio of $\geq 20 \mathrm{pix}^{-1}$ could be obtained with $\sim 2$ hour exposures.

Table 1 summarizes the properties of our sample quasars including coordinates, emission redshift, optical magnitude, bolometric luminosity, and radio-loudness.

\section{NEW OBSERVATIONS}

We observed the four quasars noted in the previous section with the Subaru Telescope and High Dispersion Spectrograph (HDS) on 2016 January 27. The weather conditions were very good throughout the night with a typical seeing of $\sim 0$ !. 6 . The time delays from the first observations of those quasars are $\sim 15-20$ years in the observed frame. We acquired high-resolution spectra $(R$ $\sim 45,000$ ) with a slit width of $0 !^{\prime \prime} 8$ and adopted bins of 2 pixels in both the spatial and dispersion directions (i.e., $\sim 0.03 \AA$ per pixel) to increase the $\mathrm{S} / \mathrm{N}$ ratio. To cover as many NALs as possible, we used standard setups, StdYa for Q0450-1310 and HE1009+2956 and Std-Yc for HE0940-1050 and Q1107+4847. These cover a wavelength range of $4030-4800 \AA$ or $4420-5660 \AA$ on the blue CCD and 4940-5660 $\AA$ or $5860-7050 \AA$ on the red CCD, respectively. We took four 1800s exposures for each quasar (i.e., a total integration time of 7200s), except for Q0450-1310 whose total integration time was $10800 \mathrm{~s}(1800 \mathrm{~s} \times 6)$.

We reduced the data in a standard manner with the IRAF $^{8}$ software package. Wavelength calibration was performed with the help of spectra of a Th-Ar lamp. We fitted the effective continuum, which also includes a substantial contribution from broad emission lines, with a third-order cubic spline function to normalize the spectra. We did not adjust the wavelength resolution of the new spectra to match that of the old spectra, because the typical line width of absorption components is large enough ( $b \geq 10 \mathrm{~km} \mathrm{~s}^{-1}$; Misawa et al. 2007a) that they are fully resolved. The final $\mathrm{S} / \mathrm{N}$ ratio around $\sim 4900 \AA$ is $\sim 15-47$ per pixel for each quasar. The log of our new and old observations is given in Table 1, including observation date, spectral resolution, total integration time, and $\mathrm{S} / \mathrm{N}$ ratio per pixel. In the same table we also give the rest-frame time interval between the observations.

\section{CROSS-CORRELATION ANALYSIS}

We measured velocity shifts by comparing NAL profiles in the first and second epochs, using the cross-correlation function method that was originally adopted for BALs in Grier et al. (2016).

We first isolated a spectral region around each NAL with $\sim 2 \AA$ margins on both sides $^{9}$, and resampled it to bins of $0.05 \AA$, which is the typical dispersion (pixel scale) of all spectra in our sample (see Figure 1). Next, we normalized the clipped spectrum using a low-order spline function avoiding absorption regions. Finally, we used the spectra from the first and second epochs to calculate Pearson's cross-correlation coefficient,

$$
r=\frac{\sum\left(f_{1}(\lambda)-\overline{f_{1}(\lambda)}\right)\left(f_{2}(\lambda)-\overline{f_{2}(\lambda)}\right)}{\sqrt{\sum\left(f_{1}(\lambda)-\overline{f_{1}(\lambda)}\right)^{2}} \sqrt{\sum\left(f_{2}(\lambda)-\overline{f_{2}(\lambda)}\right)^{2}}},
$$

where $f_{1}(\lambda)$ and $f_{2}(\lambda)$ are the normalized flux densities in the first and second epochs and $\overline{f_{1}(\lambda)}$ and $\overline{f_{2}(\lambda)}$ are their average values. We repeated the calculation of $r$ after shifting one spectrum from -15 pixel to +15 pixel in steps of $0.05 \AA$ to build the Cross Correlation Function (CCF). From the CCF we measured the velocity shift corresponding to the CCF centroid, $\Delta v(\mathrm{CCF})$, by finding the weighted center of the CCF using the portion corresponding to $r>0.8 r_{\text {peak }}$, where $r_{\text {peak }}$ is the peak value of the CCF. We performed this analysis separately for the blue and red members of doublets (i.e., C IV $\lambda \lambda 1548,1551$, Si IV $\lambda \lambda 1394,1403$, and $\mathrm{N} v \lambda \lambda 1239,1243)$, if both were detected. As an example, the CCF for the class-B Si IV

\footnotetext{
8 IRAF is distributed by the National Optical Astronomy Observatories, which are operated by the Association of Universities for Research in Astronomy, Inc., under cooperative agreement with the National Science Foundation.

${ }^{9}$ We require at least $\pm 1 \AA$ on both sides even if the $\pm 2 \AA$ regions are partially affected by data defects or line blending.
} 
NAL at $z_{\mathrm{abs}}=2.8347$ for HE0940-1050 is shown in Figure 2. The $\Delta v(\mathrm{CCF})$ values for all the NALs are summarized in Table 2 .

To determine the uncertainties, we performed a Monte Carlo simulation using synthetic spectra in which the flux density of each pixel was perturbed by a random amount drawn from a Gaussian distribution with a mean equal to the measured flux density and a standard deviation equal to the uncertainty. We constructed 100 synthetic spectra for each epoch, and repeated the CCF calculations 10,000 times using all combinations of the artificial spectra in both epochs (i.e., $100 \times 100)$. We measured the average of the CCF centroid (Cross-Correlation Centroid Distribution; $\Delta v(\mathrm{CCCD}))$ and its $1 \sigma$ uncertainty for each NAL, and we summarize these in Table 2.

To assess the performance of the CCF and CCCD methods used above, we carried out the following tests

1. We investigated whether the sampling of the spectra led to systematic errors in the shift determination. We synthesized two normalized spectra corresponding to those in the first and second epochs, in which we added a single component of a $\mathrm{C}$ IV $\lambda 1548$ line at $z_{\mathrm{abs}}=2.2367$ and 2.236722 , respectively. Both spectra have a wavelength range of $\lambda=5007.5-5014.5 \AA$. The velocity offset between the $\mathrm{C}$ IV lines ${ }^{10}$ is $2.03 \mathrm{~km} \mathrm{~s}^{-1}$. We used common line parameters except for redshift: column density and Doppler parameter of $\log \left(N_{\mathrm{C}} \mathrm{IV} / \mathrm{cm}^{-2}\right)=13.5$ and $b=12.0 \mathrm{~km} \mathrm{~s}^{-1}$ (i.e., typical values of our NAL sample), and a coverage fraction ${ }^{11}$ of $C_{\mathrm{f}}=1.0$ (i.e., a full coverage). We also assumed a wavelength resolution of $R=45,000$ (a typical value of our data), a dispersion of $\Delta \lambda=0.01 \AA$ per pixel, and finally added a noise corresponding to an uncertainty of 0.033 in each pixel (i.e., S/N ratio $\sim 30 \mathrm{pixel}^{-1}$ ).

First, we constructed synthetic spectra for a range of different dispersions, from $\Delta \lambda=0.01 \AA$ pixel $^{-1}$ to $0.15 \AA$ pixel $^{-1}$ in steps of $0.01 \AA$ pixel $^{-1}$, and used them to perform the CCF and CCCD analyses and evaluate the shift between them. We also performed the same test on an observed spectrum, the C IV $\lambda 1548$ line at $z_{\text {abs }}=2.2316$ for HE01304021 , for which we found a velocity shift of $\Delta v=$ $1.34 \mathrm{~km} \mathrm{~s}^{-1}$ based on the default dispersion (i.e., $\Delta v=0.05 \AA)$. The results for the synthetic and the observed spectra are shown in Figure 3 as a function of dispersion. Both results are obviously affected by the wavelength bin size. The shift, $\Delta v$, first decreases slightly with increasing $\Delta \lambda$ up to $0.1 \AA$ pixel $^{-1}$ then decreases slightly around $\Delta \lambda \sim$ $0.12 \AA$ pixel $^{-1}$ and increases again towards $\Delta \lambda \sim$ $0.15 \AA$ pixel $^{-1}$. Certainly, this pattern should also

10 This is not due to the cosmic expansion, but calculated by the relativistic Doppler formula,

$$
\frac{\Delta v}{c}=-\frac{\left(1+z_{1}\right)^{2}-\left(1+z_{2}\right)^{2}}{\left(1+z_{1}\right)^{2}+\left(1+z_{2}\right)^{2}}
$$

where $z_{1}$ and $z_{2}$ are apparent redshift of the C IV absorber at the first and second epochs, respectively.

11 The fraction of photons from the background light source(s) that pass through the absorber (e.g., Wampler et al. 1995: Barlow \& Sargent 1997 depend on the spectral resolution, however, in our case (i.e., $R \sim 45,000$ ), any systematic error in the shift would be negligible since we adopt a dispersion of $\Delta \lambda<0.07 \AA$ pixel $^{-1}$ or smaller.

We then carried out the same test while changing the starting wavelength for sampling by one-fifth of the bin size (i.e., by $0.01 \AA$ ). The results are shown in Figure 4 , which illustrates that there the is no obvious systematic error.

2. We compared the results from the above methods with shift measurements made with the " $\chi^{2}$ crosscorrelation" method of Eracleous et al. (2012, see their §6.1). We also carried out simulations as described in Eracleous et al. (2012) to determine the smallest shifts measurable with the available data and verify the reliability of the uncertainties. We found the results of the two independent methods of shift measurement to be in very good agreement with each other.

From the above tests, we conclude that the $\mathrm{CCF}$ and CCCD methods, as we implement them here, are robust and are not affected by the wavelength binning scheme that we adopted. Henceforth, we adopt the results of the $\mathrm{CCF}$ and CCCD analysis, as summarized in Table 2, and we discuss them below.

We found the distributions of the $\Delta v(\mathrm{CCF})$ and $\Delta v(\mathrm{CCCD})$ to be systematically shifted from zero (see Figure (5), which we attribute to a small, systematic linear offset of the spectra in each pair resulting from an uncertainty of wavelength calibration using Th-Ar spectra. To quantify this systematic effect, we measured the velocity shift of intrinsic (i.e., class-A and B) NALs compared to intervening (i.e., class-C) NALs between the two epochs for each quasar, instead of using the values of $\Delta v(\mathrm{CCCD})$ obtained by comparing directly the intrinsic NALs from the two epochs.

We also noticed that there is a systematic non-linear distortion in relative wavelength between spectra at different epochs (e.g., Griest et al. 2010; Evans et al. 2014). For example, the $\Delta v(\mathrm{CCCD})$ values for the blue and red members of the C IV NAL at $z_{\text {abs }}=1.6968$ toward Q0450-1310 (i.e., $-4.26 \pm 1.40$ and $-0.91 \pm 2.53$ ) do not match, although they should be the same. To evaluate the distortion error ${ }^{12}$, we compared all pairs of intervening (class-C) lines and examined the distribution of velocity shift differences (i.e., $|\Delta v|_{i j}=\mid \Delta v(\mathrm{CCCD})_{i}-$ $\Delta v(\mathrm{CCCD})_{j} \mid$ between $i$-th and $j$ th lines in a same spectrum) and calculate their root mean square (r.m.s.) values as summarized in Table 3. For example, we detect 14 class-C lines (here, we double-count lines if both blue and red members of a doublet are detected) in HE0940 1050 , and calculate $|\Delta v|$ for 91 pairs (i.e., ${ }_{14} C_{2}$ ) in total. The velocity difference and its total uncertainty, $\Delta v$ and $\sigma(\Delta v)$, for each line, in which we add errors from the CCCD analysis and the non-linear distortion in quadrature, are included in Table 2

Our sample quasars have multiple NALs in their spectra (from 3 NALs in HE1009+2956 to 10 NALs in HE0940-1050). Therefore, we also determined a

12 Here, the distortion error should depend not only on wavelength calibration but on data quality i.e., the $\mathrm{S} / \mathrm{N}$ ratio. 
weighted mean value of $\Delta v$ and its uncertainty for all the class-A+B NALs in each quasar and, separately, all the class-C NALs in each quasar using the following equations,

$$
\Delta v=\frac{\sum_{\mathrm{i}=1}^{\mathrm{n}}\left(\frac{1}{\sigma\left(\Delta v_{i}\right)^{2}}\right) \Delta v_{i}}{\sum_{\mathrm{i}=1}^{\mathrm{n}}\left(\frac{1}{\sigma\left(\Delta v_{i}\right)^{2}}\right)}
$$

and

$$
\sigma(\Delta v)=\sqrt{\frac{\sum_{\mathrm{i}=1}^{\mathrm{n}}\left(\frac{1}{\sigma\left(\Delta v_{i}\right)^{2}}\right)\left(\Delta v_{\mathrm{i}}-\overline{\Delta v}\right)^{2}}{(n-1) \sum_{\mathrm{i}=1}^{\mathrm{n}}\left(\frac{1}{\sigma\left(\Delta v_{i}\right)^{2}}\right)}}
$$

where $n$ is the number of absorption lines in a quasar and $\Delta v_{i}$ and $\sigma\left(\Delta v_{i}\right)$ are the velocity shift and its uncertainty between the observations for the $i$ th line of a given quasars, and $\overline{\Delta v}$ is their average. (i.e., $\sum_{\mathrm{i}=1}^{\mathrm{n}} \Delta v_{i} / n$ ). If $n<2$, as in the class-A/B NALs in HE1009+2956 and Q1107+4847, we take only the statistical error into consideration and, instead of using equation 4, we calculate $\sigma(\Delta v)$ as

$$
\sigma(\Delta v)=\sqrt{\frac{1}{\sum_{\mathrm{i}=1}^{\mathrm{n}}\left(\frac{1}{\sigma\left(\Delta v_{i}\right)^{2}}\right)}} .
$$

We also perform the same calculation using only reliable NALs after removing those that i) show line-locking, ii) are partially affected by line blending or have a data defect within $2 \AA$ of a NAL, or iii) show $>4 \sigma$ variability in their equivalent width or line profile. The resulting average $\Delta v$ and its uncertainty, $\sigma(\Delta v)$, for all the class$\mathrm{A}+\mathrm{B}$ and the class-C NALs in the quasars of our sample are shown in Figure 5 and tabulated in Table 4.

\section{RESULTS}

We applied the CCF and the CCCD analyses to 56 lines $^{13}$ in 40 NALs detected in six optically bright quasars. For the two NALs that exhibit line-locking ${ }^{14}$, we applied the analyses to the blue and red members of the doublets simultaneously. The velocity plots of the NALs are shown in Figure 1, except for the C IV NAL at $z_{\mathrm{abs}}=2.7243$ in $\mathrm{Q} 1107+4847$ because it is severely affected by data defect in the second spectrum.

As an example, we show the absorption profile of the blue member of the class-B Si IV NAL (i.e., Si IV $\lambda 1394$ ) at $z_{\mathrm{abs}}=2.8347$ in HE0940-1050, and the results of the $\mathrm{CCF}$ and CCCD analyses in Figure 2, Below we describe in detail the results for each quasar. The results of the $\mathrm{CCF}$ and the CCCD analyses for each NAL and each quasar are summarized in Table 2 and Table 4. The weighted averages of $\Delta v$ for class- $\mathrm{A}+\mathrm{B}$ and $\mathrm{C}$ NALs are also shown in Figures 5 .

HE0130-4021 Misawa et al. (2007a) detected six NALs (4 C IV, $1 \mathrm{Si}$ IV, and $1 \mathrm{~N} \mathrm{~V}$ ), of which three are classified as class-A/B NALs. We observed

\footnotetext{
13 Here, we count the two lines of a doublet separately, if they are both clearly detected without any line blending or data defects.

14 The red member of a doublet is aligned with the blue member of the following doublet. This is one of the indicators that a NAL is physically associated with a quasar (Arav 1996).
}

all of these twice with a time interval of $\Delta t_{\mathrm{obs}}$ $\sim 11.7$ yrs in the observed frame, corresponding to $\Delta t_{\text {rest }} \sim 2.90$ yrs in the quasar rest frame. Among the 12 blue/red lines of the six NALs, seven are detected without being affected by data defect or other unrelated lines. The wavelength margin is smaller than $2 \AA$ around the $\mathrm{N}$ V $\lambda 1239$ line at $z_{\mathrm{abs}}=2.9749$. The profiles of the Si IV $\lambda 1394$ line at $z_{\mathrm{abs}}=2.8570$ and the $\mathrm{N}$ v $\lambda 1243$ line at $z_{\text {abs }}=2.9749$ show a hint of time variability (see Figure 1). The weighted average velocity shift for class-A/B NALs $\left(\Delta v_{\mathrm{AB}}=0.45 \pm 0.32 \mathrm{~km} \mathrm{~s}^{-1}\right)$ is not significantly shifted from that for class-C NALs $\left(\Delta v_{\mathrm{C}}=1.28 \pm 0.50 \mathrm{~km} \mathrm{~s}^{-1}\right)$. Even if we consider only the reliable NALs (2 class-A/B lines and 2 class-C lines) that are classified as a1 in Table 2. the difference would still be insignificant. We conclude that there is no clear velocity shift of the class-A/B NALs between our observations.

Q0450-1310 In the spectrum of this radio-quiet quasar, Misawa et al. (2007a) detected 10 NALs (5 C IV and $5 \mathrm{Si}$ IV), of which only the $\mathrm{C}$ IV NAL at $z_{\text {abs }}$ $=2.2307\left(v_{\mathrm{ej}} \sim 6370 \mathrm{~km} \mathrm{~s}^{-1}\right)$ is classified as intrinsic (class-A). Because two C IV NALs at $z_{\text {abs }}$ $=2.1050$ and 2.1061, and three Si IV NALs at $z_{\text {abs }}=2.1051,2.1062$, and 2.1069 are close to each other within $\sim 170 \mathrm{~km} \mathrm{~s}^{-1}$, we treat them as single NALs $\left(\mathrm{C}\right.$ IV NAL at $z_{\text {abs }} \sim 2.1061$ and Si IV NAL at $\left.z_{\text {abs }} \sim 2.1059\right)$, respectively. After removing NALs that are blended with data defects or other unrelated lines, we are left with 10 lines in 6 NALs. The time interval between the observations is $\Delta t_{\text {obs }} \sim 17.1$ yrs (i.e., $\Delta t_{\text {rest }} \sim 5.2 \mathrm{yrs}$ ). The weighted average velocity shift of the class-A NAL $\left(\Delta v_{\mathrm{AB}}=-3.30 \pm 0.38 \mathrm{~km} \mathrm{~s}^{-1}\right)$ is not shifted significantly from that of class-C NALs $\left(\Delta v_{\mathrm{C}}=\right.$ $\left.-3.87 \pm 0.30 \mathrm{~km} \mathrm{~s}^{-1}\right)$. Even after we consider only reliable NALs ( 2 class-A lines and 6 class- $\mathrm{C}$ lines) that satisfy all the criteria (see $\S 4$ ), the difference is not significant. We conclude that the class-A NAL is not significantly shifted between our observations.

HE0940-1050 In total, 10 NALs (7 C IV and $3 \mathrm{Si}$ IV) were detected, of which only one Si IV NAL at $z_{\text {abs }}=2.8347\left(v_{\mathrm{ei}} \sim 18600 \mathrm{~km} \mathrm{~s}^{-1}\right)$ is classified as class-B (Misawa et al. 2007a). No class-A NALs were identified. Although the C IV NAL at $z_{\text {abs }}=$ 2.8346 was originally classified as class-C, it could be intrinsic to the quasar because i) it has a similar redshift to the class-B Si IV NAL and because ii) it shows line-locking with a $\mathrm{C}$ IV NAL at $z_{\text {abs }}$ $=2.8245$. We regard the two line-locked C IV NALs as a single NAL at $z_{\text {abs }} \sim 2.8294$. After removing NALs that are blended with data defects or other unrelated lines, we are left with 17 lines in 10 NALs. The time interval between the observations is $\Delta t_{\text {obs }} \sim 15.8$ yrs (i.e., $\Delta t_{\text {rest }} \sim$ $3.9 \mathrm{yrs})$. The weighted average velocity shift of class-A/B NALs $\left(\Delta v_{\mathrm{AB}}=-1.51 \pm 0.37 \mathrm{~km} \mathrm{~s}^{-1}\right)$ is not clearly shifted relative to that of class-C NALs $\left(\Delta v_{\mathrm{C}}-0.94 \pm 0.12 \mathrm{~km} \mathrm{~s}^{-1}\right)$. Even if we consider only reliable NALs (2 class-B lines and 12 class- 
$\mathrm{C}$ lines), the difference would still be insignificant. Thus, we conclude that the class-B NAL does not show a velocity shift between our observations compared to class-C NALs.

HE1009+2956 Because of limited wavelength coverage, Misawa et al. (2007a) detected only 3 NALs (1 C IV, $1 \mathrm{Si}$ IV, and $1 \mathrm{~N} \mathrm{v}$ ), of which a Si IV NAL at $z_{\text {abs }}=2.2533\left(v_{\mathrm{ej}} \sim 33900 \mathrm{~km} \mathrm{~s}^{-1}\right)$ and a N v NAL at $z_{\text {abs }}=2.6495\left(v_{\mathrm{ej}} \sim-450 \mathrm{~km} \mathrm{~s}^{-1}\right)$ are classified as class-A. Unfortunately, we cannot monitor the variability of the $\mathrm{N} v$ NAL profile because of blending with other lines and a data defect in the first spectrum. We used only three lines in two NALs for our analyses. The time interval between the observations is $\Delta t_{\text {obs }} \sim 20.1$ yrs (i.e., $\Delta t_{\text {rest }} \sim$ $5.5 \mathrm{yrs})$. The weighted average of the velocity shift of class-A/B NALs $\left(\Delta v_{\mathrm{AB}}=-1.67 \pm 1.07 \mathrm{~km} \mathrm{~s}^{-1}\right)$ is not significantly shifted compared to the class-C NALs $\left(\Delta v_{\mathrm{C}}-0.68 \pm 0.49 \mathrm{~km} \mathrm{~s}^{-1}\right)$. All detected NALs satisfy the criteria to be in our reliable sample. Thus, we conclude that there is no clear velocity shift of the class-A Si IV NAL between our observations. For the quasar, we used equation 5 to calculate the weighted average of the velocity shift for the class-A NAL because the sample includes only one member.

Q1107+4847 Misawa et al. (2007a) detected 6 NALs (3 $\mathrm{C}$ IV and $3 \mathrm{Si}$ IV), of which only one Si IV NAL at $z_{\mathrm{abs}}=2.7243\left(v_{\mathrm{ej}} \sim 21400 \mathrm{~km} \mathrm{~s}^{-1}\right)$ is classified as class-A. There are no class-B NALs. A $\mathrm{C}$ IV NAL at $z_{\mathrm{abs}}=2.7243$, originally classified as class-C, also could be intrinsic to the quasar because it has the same redshift as the class-A Si IV NAL. Although all 6 NALs are covered by our spectra, four of the lines, including the C IV NAL at $z_{\text {abs }}=2.7243$, are affected by data defects or are severely blended with other lines. As a result, we used 8 lines in 5 NALs for the CCF and the CCCD analyses. The time interval between the observations is $\Delta t_{\mathrm{obs}} \sim 20.7$ yrs that corresponds to $\Delta t_{\text {rest }} \sim 5.2$ yrs. The velocity shift of the class-A $\operatorname{NAL}\left(\Delta v_{\mathrm{AB}}=-5.84 \pm 1.56 \mathrm{~km} \mathrm{~s}^{-1}\right)$ is not obviously shifted compared to the class-C NALs $\left(\Delta v_{\mathrm{C}}\right.$ $\left.=-3.38 \pm 0.39 \mathrm{~km} \mathrm{~s}^{-1}\right)$. The significance level becomes smaller when we remove NALs that do not satisfy all the selection criteria. Because all NALs for the quasar are blueshifted significantly (i.e., $\Delta v \leq-3 \mathrm{~km} \mathrm{~s}^{-1}$ ), we speculate that the class-C NALs are also intrinsic to the quasar and accelerated with the class-A NAL. Indeed, some intrinsic NALs could have full coverage (i.e., classified into class-C) if absorbing clouds in the vicinity of the continuum source are large enough to cover it completely (Misawa et al. 2007a). To test the idea, we performed the CCF and CCCD analyses to a $\mathrm{Mg}$ II $\lambda 2803$ line at $z_{\mathrm{abs}}=0.8076$ whose origin should be unrelated to the outflowing winds because its offset velocity would be $>0.66 \mathrm{c}$ if we assume it is an intrinsic NAL. We derived an offset velocity of $\Delta v=-5.61 \pm 0.28 \mathrm{~km} \mathrm{~s}^{-1}$ for the $\mathrm{Mg}$ II NAL that is close to those of class-A/B and class-C NALs. Thus, we do not find any significant velocity shifts between our observations. Here, we used equation 5 to calculate the weighted average of the velocity shift for the class-A NAL because the sample includes only one member.

HS1700 +6416 This extremely bright quasar could be amplified by gravitational lensing by two clusters of galaxies along our sight-line. Misawa et al. (2007a) detected 10 NALs (6 C IV, $2 \mathrm{Si}$ IV, and $2 \mathrm{~N} \mathrm{~V}$ ), of which three C IV NALs at $z_{\text {abs }}=2.4330,2.4394$, and 2.7125 , and two $\mathrm{N}$ v NALs at $z_{\text {abs }}=2.7125$ and 2.7164 are classified as class-A or B. A C IV NAL at $z_{\text {abs }}=2.1680$ is not covered by our secondepoch spectrum. Because two C IV NALs at $z_{\text {abs }}=$ 2.4330 and 2.4394 show line-locking, we treat them as a single NAL at $z_{\mathrm{abs}} \sim 2.4390$. Thus, we used 11 lines in 8 NALs for the analyses. The time interval of the observations is $\Delta t_{\text {obs }} \sim 10.3$ yrs (i.e., $\Delta t_{\text {rest }} \sim$ $2.8 \mathrm{yrs}$ ). The weighted average velocity shift of the class-A/B NALs $\left(\Delta v_{\mathrm{AB}}=1.12 \pm 0.45 \mathrm{~km} \mathrm{~s}^{-1}\right)$ is not significantly shifted from that for class-C NALs $\left(\Delta v_{\mathrm{C}}=0.56 \pm 0.31 \mathrm{~km} \mathrm{~s}^{-1}\right)$. Even if we consider only the reliable NALs (3 class-A lines and 5 classC lines) that satisfy all the selection criteria, the significance level would still be insignificant. We conclude that we do not detect a velocity shift between our observations.

The main result of the CCF and the CCCD analyses is that we do not detect significant weighted average velocity shifts of class-A/B relative to class-C NALs, placing a $3 \sigma$ upper limit on the acceleration/deceleration of intrinsic NALs with a magnitude of $\sim 0.7 \mathrm{~km} \mathrm{~s}^{-1} \mathrm{yr}^{-1}$ $\left(\sim 0.002 \mathrm{~cm} \mathrm{~s}^{-2}\right)$ in average.

\section{DISCUSSION}

We monitored intrinsic NALs in 6 quasars for 2.8$5.5 \mathrm{yr}$ in the quasar rest frame. We found no highly significant shifts in NAL velocities, implying a $3 \sigma$ upper limits on the acceleration of $\sim 0.7 \mathrm{~km} \mathrm{~s}^{-1} \mathrm{yr}^{-1}$ $\left(\sim 0.002 \mathrm{~cm} \mathrm{~s}^{-2}\right)$. The limits on NAL acceleration found here appear to be two or three orders of magnitude lower than those of BALs reported in the literature (Joshi et al. 2018; Grier et al. 2016; J Joshi et al. 2014; Hall et al.|2007; Gabel et al.|2003; Rupke et al.|2002; Vilkoviskij \& Irwin 2001). This is particularly noteworthy since the NAL quasars in our sample have larger bolometric luminosities than the BAL quasars reported in the literature.

The simplest hypothesis for the acceleration of NAL systems invokes radiation pressure. Thus, the equation of motion can be cast as

$$
\frac{d v}{d t}=\frac{f L}{4 \pi r^{2} c m_{p} N_{H}}-\frac{G M}{r^{2}},
$$

where $v$ is the outflow velocity, $L$ is the quasar's bolometric luminosity, $f$ is the fraction of the luminosity that contributes to the acceleration, $r$ is a distance from the flux source, $N_{H}$ is the total column density of the absorber, and $M$ is the mass of the central black-hole (Hamann 1998). By considering the typical luminosity and black hole mass of our target quasars (i.e., $L$ $\left.=10^{48} \mathrm{erg} \mathrm{s}^{-1}, M=10^{9} M_{\odot}\right)$ as well as a typical hydrogen column density of NAL absorbers $\left(N_{H}=10^{18} \mathrm{~cm}^{-2}\right.$; 
e.g., Wu et al. 2010), equation 6 becomes

$$
\begin{aligned}
\Delta v \sim & 52 f \cos \theta\left(\frac{\Delta t}{1 \mathrm{yr}}\right)\left(\frac{r}{1 \mathrm{kpc}}\right)^{-2} \\
& \times\left(\frac{L}{10^{48} \mathrm{erg} \mathrm{s}^{-1}}\right)\left(\frac{N_{H}}{10^{18} \mathrm{~cm}^{-2}}\right)^{-1} \mathrm{~km} \mathrm{~s}^{-1},
\end{aligned}
$$

where we ignore gravity because its contribution is much smaller than the radiative force as long as $f>10^{-7}$. We also include a factor of $\cos \theta$, where $\theta$ is the angle between the outflow stream lines and our sightline.

The implied acceleration of intrinsic NALs for our 6 quasars is smaller than the prediction of equation [6 by a factor of 100 or more, if we assume a distance of NAL absorbers from the central engine of $r \sim 1 \mathrm{kpc}$, following Arav et al. (2013), for example. Thus, if radiation pressure is the main acceleration mechanism, we infer that, either $f \cos \theta \leq 10^{-2}$, or $r>10 \mathrm{kpc}$, or both (see Figure 6). Because reports of absorber distances as large as $10 \mathrm{kpc}$ are uncommon in the literature, we speculate that one or a combination of the following is the cause for the small inferred accelerations: (i) $f<10^{-2}$, which is much smaller than the value suggested for X-ray Ultra Fast Outflows ( $f \geq 0.05$; e.g., Tombesi et al. 2013), (ii) Our sightline toward the flux source is almost perpendicular to some of the outflow stream lines as suggested in Arav et al. (1999), (iii) The acceleration is episodic and it is only observable in a small fraction of quasars at any given time. The last hypothesis is motivated by the results of Grier et al. (2016) who were able to detect an acceleration in only three out of 140 quasars and noted that the acceleration was not constant in time. If this is the case for NALs as well, we would need to observe a much larger sample of objects or make many repeated observations of this sample in order to detect NAL acceleration.

\section{SUMMARY}

In this study, we used high-resolution spectra of 6 NAL quasars that were obtained with 8-10 meter telescopes (i.e., Subaru, Keck, and VLT) at two epochs separated by $\Delta t_{\text {obs }} \sim 10.3-20.7$ years (i.e., $\Delta t_{\text {rest }} \sim 2.8-5.5$ years) to monitor velocity changes of intrinsic NALs. Our main results are as follows.

- Using the CCF and CCCD analyses, we discovered no significant acceleration/deceleration of intrinsic NALs, placing a $3 \sigma$ upper limit on the magnitude of the acceleration of $\sim 0.7 \mathrm{~km} \mathrm{~s}^{-1} \mathrm{yr}^{-1}$ $\left(\sim 0.002 \mathrm{~cm} \mathrm{~s}^{-2}\right)$.
- This magnitude of NAL acceleration is more than two orders of magnitude smaller than the expected value from radiative acceleration, which suggests that, if our sightline is almost parallel to the streamlines of the outflow, the fraction of the luminosity that contributes to the acceleration is $f<0.01$, or the absorber's distance from the flux source is greater than $10 \mathrm{kpc}$. We do not find any correlations between gas acceleration and bolometric luminosity either in BAL or in NAL quasars.

- Because of the very small velocity shifts, the NAL absorbers studied here may be at or close to their terminal velocities, i.e., they may be located at a large distance from the quasar central engine. Other possible explanations are that we observe a standing-flow with streamlines that are almost perpendicular to our sightline and gas is continuously replenished as suggested by hydrodynamical simulations (Arav et al. 1999; Proga et al. 2000), or that the acceleration process is episodic so that only a small fraction of NALs exhibit acceleration at any one time.

If the typical magnitude of NAL acceleration is close to the $3 \sigma$ upper limit we have obtained (i.e., $\sim 0.7 \mathrm{~km} \mathrm{~s}^{-1} \mathrm{yr}^{-1}$ ), we may be able to detect obvious velocity shifts in future observations compared to our first epoch spectra taken in 1995-2000. For example, intrinsic NALs are expected to be blueshifted by more than $2 \mathrm{~km} \mathrm{~s}^{-1}$ with a significance level of $\geq 3 \sigma$ in 40 years from now. Alternatively, if the acceleration process is episodic, we may be able to detect acceleration in a few quasars if we observe a sample that is an order of magnitude larger than the sample we have used here. Such a precise spectroscopic observation will also be very important to other areas of research, such as a direct measurement of cosmic expansion (e.g., Sandage 1962; Loeb 1998; Balbi \& Quercellini 2007; Liske et al. 2008) and a measurement of changes in the fine structure constant (e.g., Murphv et al. 2003; Chand et al. 2004; Srianand et al. 2004; Webb et al. 2011) using extremely large (30-m class) telescopes in coming decades.

The research was supported by the Japan Society for the Promotion of Science through Grants-in-Aid for Scientific Research 18K03698 and partially supported by a MEXT Grant-in-Aid for Scientific Research on Innovative Areas (No.15H05894).

\section{REFERENCES}

\footnotetext{
Arav, N., Borguet, B., Chamberlain, C., Edmonds, D., \& Danforth, C. 2013, MNRAS, 436, 3286

Arav, N., Korista, K. T., de Kool, M., Junkkarinen, V. T., \& Begelman, M. C. 1999, ApJ, 516, 27

Arav, N. 1996, ApJ, 465, 617

Balbi, A., \& Quercellini, C. 2007, MNRAS, 382, 1623

Balsara, D. S., \& Krolik, J. H. 1993, ApJ, 402, 109

Barlow, T. A., \& Sargent, W. L. W. 1997, AJ, 113, 136

Bruni, G., Mack, K.-H., Salerno, E., et al. 2012, A\&A, 542, A13

Capellupo, D. M., Hamann, F., Shields, J. C., Rodríguez Hidalgo, P., \& Barlow, T. A. 2011, MNRAS, 413, 908

Capellupo, D. M., Hamann, F., Shields, J. C., Rodríguez Hidalgo, P., \& Barlow, T. A. 2012, MNRAS, 422, 3249
}

Chand, H., Srianand, R., Petitjean, P., \& Aracil, B. 2004, A\&A, 417, 853

Eracleous, M., Boroson, T. A., Halpern, J. P., \& Liu, J. 2012, ApJS, 201, 23

Evans, T. M., Murphy, M. T., Whitmore, J. B., et al. 2014 MNRAS, 445, 128

Everett, J. E. 2005, ApJ, 631, 689

Filiz Ak, N., Brandt, W. N., Hall, P. B., et al. 2012, ApJ, 757, 114

Fukumura, K., Kazanas, D., Contopoulos, I., \& Behar, E. 2010, ApJ, 723, L228

Gabel, J. R., Crenshaw, D. M., Kraemer, S. B., et al. 2003, ApJ, 595,120

Gallagher, S. C., Brandt, W. N., Wills, B. J., et al. 2004, ApJ, 603,425 
Gibson, R. R., Brandt, W. N., Schneider, D. P., \& Gallagher, S. C. 2008, ApJ, 675, 985-1001

Grier, C. J., Brandt, W. N., Hall, P. B., et al. 2016, ApJ, 824, 130

Griest, K., Whitmore, J. B., Wolfe, A. M., et al. 2010, ApJ, 708, 158

Hall, P. B., Brandt, W. N., Petitjean, P., et al. 2013, MNRAS, 434, 222

Hall, P. B., Sadavoy, S. I., Hutsemekers, D., Everett, J. E., \& Rafiee, A. 2007, ApJ, 665, 174

Hall, P. B., Anderson, S. F., Strauss, M. A., et al. 2002, ApJS, 141,267

Hamann, F. 1998, ApJ, 500, 798

Hamann, F., Kaplan, K. F., Rodríguez Hidalgo, P., Prochaska, J. X., \& Herbert-Fort, S.' 2008, MNRAS, 391, L39

Hamann, F., Simon, L., Rodriguez Hidalgo, P., \& Capellupo, D. 2012, AGN Winds in Charleston, 460, 47

Joshi, R., Srianand, R., Chand, H., et al. 2018, arXiv:1808.05622

Joshi, R., Chand, H., Srianand, R., \& Majumdar, J. 2014, MNRAS, 442, 862

Kellermann, K.I., Sramek, R., Schmidt, M., Shaffer, D.B., \& Green, R., 1989, AJ, 98, 119

Kellermann, K.I., Sramek, R.A., Schmidt, M., Green, R.F., \& Shaffer, D.B., 1994, AJ, 108, 1163

Leighly, K. M., Terndrup, D. M., Baron, E., et al. 2014, ApJ, 788, 123

Lípari, S. L., \& Terlevich, R. J. 2006, MNRAS, 368, 1001

Liske, J., Grazian, A., Vanzella, E., et al. 2008, MNRAS, 386, 1192

Loeb, A. 1998, ApJ, 499, L111

Misawa, T., Charlton, J. C., Eracleous, M., et al. 2007, ApJS, 171,1

Misawa, T., Eracleous, M., Charlton, J. C., \& Kashikawa, N. 2007, ApJ, 660, 152
Misawa, T., Charlton, J. C., \& Eracleous, M. 2014, ApJ, 792, 77 Murphy, M. T., Webb, J. K., \& Flambaum, V. V. 2003, MNRAS, 345,609

Murray, N., Chiang, J., Grossman, S. A., \& Voit, G. M. 1995, ApJ, 451, 498

Proga, D., Stone, J. M., \& Kallman, T. R. 2000, ApJ, 543, 686 Richards, G. T., Lacy, M., Storrie-Lombardi, L. J., et al. 2006, ApJS, 166, 470

Rupke, D. S., Veilleux, S., \& Sanders, D. B. 2002, ApJ, 570, 588

Sandage, A. 1962, ApJ, 136, 319

Springel, V., Di Matteo, T., \& Hernquist, L. 2005, ApJ, 620, L79

Srianand, R., Chand, H., Petitjean, P., \& Aracil, B. 2004,

Physical Review Letters, 92, 121302

Tombesi, F., Cappi, M., Reeves, J. N., et al. 2013, MNRAS, 430 1102

Vilkoviskij, E. Y., \& Irwin, M. J. 2001, MNRAS, 321, 4

Vivek, M., Srianand, R., Mahabal, A., \& Kuriakose, V. C. 2012, MNRAS, 421, L107

Wampler, E. J., Chugai, N. N., \& Petitjean, P. 1995, ApJ, 443 586

Webb, J. K., King, J. A., Murphy, M. T., et al. 2011, Physical Review Letters, 107, 191101

Weymann, R. J., Morris, S. L., Gray, M. E., \& Hutchings, J. B. 1997, ApJ, 483, 717

Weymann, R. J., Morris, S. L., Foltz, C. B., \& Hewett, P. C. 1991, ApJ, 373, 23

Wu, J., Charlton, J. C., Misawa, T., Eracleous, M., \& Ganguly, R. 2010, ApJ, 722, 997 
TABLE 1

SAMPLE QUASARS

\begin{tabular}{|c|c|c|c|c|c|c|c|c|c|c|c|c|}
\hline Quasar & $\begin{array}{c}\text { RA } \\
\text { (hh } \mathrm{mm} \mathrm{ss})\end{array}$ & $\begin{array}{c}\text { Dec } \\
(\mathrm{dd} \mathrm{mm} \text { ss })\end{array}$ & $z_{\mathrm{em}}$ & $\begin{array}{c}m_{\mathrm{V}} \\
(\mathrm{mag})\end{array}$ & $\begin{array}{c}L_{\mathrm{bol}^{a}} \\
\left(\operatorname{ergs~s}^{-1}\right)\end{array}$ & $R^{b}$ & Obs Date & Tel./Inst. & $R$ & $\begin{array}{l}T_{\exp } \\
(\mathrm{sec})\end{array}$ & $\begin{array}{c}\mathrm{S} / \mathrm{N}^{c} \\
\left(\text { pixel }^{-1}\right)\end{array}$ & $\begin{array}{c}\Delta t_{\text {rest }}{ }^{d} \\
(\mathrm{yrs})\end{array}$ \\
\hline HE0130-4021 & 013302 & -400628 & 3.030 & 17.02 & $1.1 \times 10^{48}$ & 11.2 & $\begin{array}{l}1995 \text { Dec. } 28 \\
2007 \text { Sep. } 5\end{array}$ & $\begin{array}{c}\text { Keck/HIRES } \\
\text { VLT/UVES }\end{array}$ & $\begin{array}{l}36000 \\
40000\end{array}$ & $\begin{array}{c}14400 \\
6000\end{array}$ & $\begin{array}{l}41 \\
46\end{array}$ & 2.9 \\
\hline Q0450-1310 & 045312 & -130546 & 2.300 & 16.50 & $1.1 \times 10^{48}$ & $<1.7$ & $\begin{array}{l}1998 \text { Dec. } 13-14 \\
\text { 2016 Jan. } 27\end{array}$ & $\begin{array}{l}\text { Keck/HIRES } \\
\text { Subaru/HDS }\end{array}$ & $\begin{array}{l}36000 \\
45000\end{array}$ & $\begin{array}{c}7200 \\
10800\end{array}$ & $\begin{array}{l}19 \\
15\end{array}$ & 5.2 \\
\hline HE0940-1050 & 094253 & -110425 & 3.080 & 16.90 & $1.3 \times 10^{48}$ & $<2.6$ & $\begin{array}{l}\text { 2000 Apr. } 3 \\
\text { 2016 Jan. } 27\end{array}$ & $\begin{array}{l}\text { VLT/UVES } \\
\text { Subaru/HDS }\end{array}$ & $\begin{array}{l}40000 \\
45000\end{array}$ & $\begin{array}{l}3600 \\
7200\end{array}$ & $\begin{array}{l}32 \\
29\end{array}$ & 3.9 \\
\hline HE1009+2956 & 101155 & +294141 & 2.644 & 16.40 & $1.5 \times 10^{48}$ & $<1.6$ & $\begin{array}{l}1995 \text { Dec. } 29 \\
\text { 2016 Jan. } 27\end{array}$ & $\begin{array}{l}\text { Keck/HIRES } \\
\text { Subaru/HDS }\end{array}$ & $\begin{array}{l}36000 \\
45000\end{array}$ & $\begin{array}{c}12200 \\
7200\end{array}$ & $\begin{array}{l}56 \\
27\end{array}$ & 5.5 \\
\hline Q1107+4847 & 111038 & +483116 & 3.000 & 16.60 & $1.6 \times 10^{48}$ & $<2.0$ & $\begin{array}{c}1995 \text { May } 9 \\
\text { 2016 Jan. } 27\end{array}$ & $\begin{array}{l}\text { Keck/HIRES } \\
\text { Subaru/HDS }\end{array}$ & $\begin{array}{l}37500 \\
45000\end{array}$ & $\begin{array}{l}7200 \\
7200\end{array}$ & $\begin{array}{l}59 \\
37\end{array}$ & 5.2 \\
\hline HS1700+6416 & 170100 & +641209 & 2.722 & 16.13 & $2.1 \times 10^{48}$ & $<1.2$ & $\begin{array}{l}1995 \text { May } 10 \\
2005 \text { Aug. } 19\end{array}$ & $\begin{array}{l}\text { Keck/HIRES } \\
\text { Subaru/HDS }\end{array}$ & $\begin{array}{l}36000 \\
36000\end{array}$ & $\begin{array}{l}11500 \\
5400\end{array}$ & $\begin{array}{l}72 \\
25\end{array}$ & 2.8 \\
\hline
\end{tabular}

a Bolometric luminosity, calculated as $L_{\mathrm{bol}}=4.4 \lambda L_{\lambda}(1450 \AA)$, following Richards et al. (2006)

b Radio-loudness, calculated as $R=f_{\nu}(5 \mathrm{GHz}) / f_{\nu}(4400 \AA)$, following Kellermann et al. $(1989$, , 1994).

c Signal to noise ratio at $\lambda_{\text {obs }} \sim 4900 \AA$

d Time interval between observations in the quasar's rest-frame. 
TABLE 2

SAMPLE NALS

\begin{tabular}{|c|c|c|c|c|c|c|c|c|c|c|}
\hline Quasar & $z_{\mathrm{abs}}$ & $\begin{array}{c}v_{\mathrm{ej}}^{a} \\
\left(\mathrm{~km} \mathrm{~s}^{-1}\right)\end{array}$ & ion & comp. ${ }^{b}$ & $\operatorname{class}^{c}$ & $\begin{array}{c}\Delta v(\mathrm{CCF})^{d} \\
\left(\mathrm{~km} \mathrm{~s}^{-1}\right)\end{array}$ & $\begin{array}{c}\Delta v(\mathrm{CCCD})^{e} \\
\left(\mathrm{~km} \mathrm{~s}^{-1}\right)^{2}\end{array}$ & $\begin{array}{c}\Delta v \pm \sigma(\Delta v)^{f} \\
\left(\mathrm{~km} \mathrm{~s}^{-1}\right)\end{array}$ & sample $^{g}$ & notes \\
\hline \multirow[t]{8}{*}{ HE0130-4021 } & 2.2316 & 65181 & C IV & $\mathrm{b}$ & B1 & & & & $\mathrm{d}$ & \multirow[t]{2}{*}{ blend with other lines. } \\
\hline & \multirow{3}{*}{2.5597} & \multirow{3}{*}{37037} & \multirow{3}{*}{ C IV } & $\mathrm{r}$ & & 1.41 & $1.34 \pm 0.34$ & $1.34 \pm 1.30$ & a1 & \\
\hline & & & & $\mathrm{b}$ & $\mathrm{A} 2$ & 0.29 & $0.26 \pm 0.21$ & $0.26 \pm 1.27$ & a1 & \multirow{3}{*}{$\begin{array}{l}\text { affected by data defect in 1st spectrum. } \\
\text { affected by data defect in 1st spectrum. }\end{array}$} \\
\hline & & & & $\mathrm{r}$ & $\mathrm{C} 2$ & & & & $\mathrm{~d}$ & \\
\hline & 2.6884 & 26503 & C IV & $\begin{array}{l}\mathrm{D} \\
\mathrm{r}\end{array}$ & 42 & 2.27 & $2.24 \pm 0.58$ & $2.24 \pm 1.38$ & $\begin{array}{l}\mathrm{a} \\
\mathrm{a} 1\end{array}$ & \\
\hline & 2.8570 & 13155 & Si IV & $\mathrm{b}$ & $\mathrm{C} 2$ & 1.19 & $1.19 \pm 0.30$ & $1.19 \pm 1.29$ & $\mathrm{c}$ & $\begin{array}{l}\text { may be time variable. } \\
\text { blend with other lines. }\end{array}$ \\
\hline & 2.8571 & 13147 & C IV & $\mathrm{b}$ & $\mathrm{C} 2$ & 0.38 & $0.49 \pm 0.44$ & $0.49 \pm 1.33$ & a1 & \multirow[b]{2}{*}{$\begin{array}{l}\text { affected by data defect in } 1 \text { st spectrum. } \\
\text { margin is }<2 \AA \text { because of data defect. } \\
\text { may be time variable. }\end{array}$} \\
\hline & 2.9749 & 4130 & N V & $\begin{array}{l}\mathrm{r} \\
\mathrm{b} \\
\mathrm{r}\end{array}$ & A2 & $\begin{array}{c}-0.16 \\
0.40\end{array}$ & $\begin{array}{c}-0.14 \pm 0.35 \\
0.34 \pm 0.49\end{array}$ & $\begin{array}{c}-0.14 \pm 1.30 \\
0.34 \pm 1.34\end{array}$ & $\begin{array}{l}\mathrm{d} \\
\mathrm{b} \\
\mathrm{c}\end{array}$ & \\
\hline \multirow[t]{10}{*}{ Q0450-1310 } & 1.6968 & 59748 & $\mathrm{C} \mathrm{IV}$ & $\mathrm{b}$ & $\mathrm{C} 2$ & -4.20 & $-4.26 \pm 1.40$ & $-4.26 \pm 2.18$ & $\mathrm{~b}$ & \multirow{4}{*}{ margin is $<2 \AA$ because of line blending. } \\
\hline & \multirow{2}{*}{1.9986} & \multirow{2}{*}{28646} & \multirow{2}{*}{ C IV } & $\mathrm{r}$ & & -1.05 & $-0.91 \pm 2.53$ & $-0.91 \pm 3.03$ & a1 & \\
\hline & & & & $\begin{array}{l}\mathrm{b} \\
\mathrm{r}\end{array}$ & $\mathrm{C} 2$ & $\begin{array}{l}-3.44 \\
-4.66\end{array}$ & $\begin{array}{l}-3.47 \pm 0.70 \\
-4.63 \pm 1.41\end{array}$ & $\begin{array}{l}-3.47 \pm 1.81 \\
-4.63 \pm 2.19\end{array}$ & $\begin{array}{l}\text { a1 } \\
\text { a1 }\end{array}$ & \\
\hline & 2.0667 & 21957 & Si IV & $\mathrm{b}$ & $\mathrm{C} 1$ & -4.10 & $-4.21 \pm 0.83$ & $-4.21 \pm 1.86$ & a1 & \\
\hline & 2.0668 & 21947 & C IV & $\begin{array}{l}\mathrm{r} \\
\mathrm{b}\end{array}$ & $\mathrm{C} 1$ & -3.67 & $-3.76+0.81$ & $-3.76+1.86$ & d & affected by data defect in 1st spectrum. \\
\hline & & & & $\mathrm{r}$ & & & & & d & affected by data defect in 1 st and 4 th spectra. \\
\hline & 2.1059 & 18163 & Si IV & $\begin{array}{l}\mathrm{b} \\
\mathrm{r}\end{array}$ & $\mathrm{C} 2$ & & & & $\mathrm{~d}$ & blend with other lines. \\
\hline & 2.1061 & 18144 & C IV & $\begin{array}{l}\mathrm{r} \\
\mathrm{b}\end{array}$ & $\mathrm{C} 1$ & -3.81 & $-3.75 \pm 0.39$ & $-3.75 \pm 1.71$ & $\begin{array}{c}\mathrm{d} \\
\mathrm{a} 1\end{array}$ & affected by data defect in 1st spectrum. \\
\hline & & & & $\mathrm{r}$ & & -4.43 & $-4.41 \pm 0.42$ & $-4.41 \pm 1.72$ & $\mathrm{~b}$ & margin is $<2 \AA$ because of data defect. \\
\hline & 2.2307 & 6366 & Si IV & $\mathrm{b}$ & A2 & -3.51 & $-3.65 \pm 0.87$ & $-3.65 \pm 1.88$ & a1 & 然 \\
\hline HE0940-1050 & 2.2210 & 69629 & C IV & $\frac{\mathrm{r}}{\mathrm{b}}$ & C1 & $\frac{-3.14}{-0.78}$ & $\begin{array}{l}-2.89 \pm 1.12 \\
-0.81 \pm 0.18\end{array}$ & $\frac{-2.89 \pm 2.01}{-0.81 \pm 0.92}$ & $\frac{\text { a1 }}{\text { a1 }}$ & \\
\hline & & & & $\mathrm{r}$ & & -1.16 & $\begin{array}{l}-1.01 \pm \pm 0.24 \\
-1.15 \pm 0.24\end{array}$ & $-1.15 \pm 0.93$ & a1 & 5. \\
\hline & 2.3302 & 60096 & C IV & b & $\mathrm{C} 1$ & -0.91 & $-0.89 \pm 0.19$ & $-0.89 \pm 0.92$ & a1 & $\Xi$ \\
\hline & & & & $\mathrm{r}$ & & -1.13 & $-1.14 \pm 0.18$ & $-1.14 \pm 0.92$ & a1 & \\
\hline & 2.4090 & 53331 & C IV & $\mathrm{b}$ & C3 & -0.95 & $-0.89 \pm 0.41$ & $-0.89 \pm 0.99$ & a1 & \\
\hline & & & & $\mathrm{r}$ & & -1.60 & $-1.62 \pm 0.73$ & $-1.62 \pm 1.16$ & a1 & \\
\hline & 2.6580 & 32625 & C IV & $\mathrm{b}$ & $\mathrm{C} 2$ & -0.95 & $-0.95 \pm 0.71$ & $-0.95 \pm 1.15$ & a1 & \\
\hline & & & & $\mathrm{r}$ & & -0.59 & $-0.48 \pm 0.54$ & $-0.48 \pm 1.05$ & a1 & \\
\hline & 2.6677 & 31839 & C IV & $\mathrm{b}$ & $\mathrm{C} 1$ & -1.07 & $-1.01 \pm 0.57$ & $-1.01 \pm 1.07$ & a1 & \\
\hline & & & & $\mathrm{r}$ & & 0.07 & $0.13 \pm 1.00$ & $0.13 \pm 1.35$ & a1 & \\
\hline & 2.6677 & 31839 & Si IV & $\mathrm{b}$ & $\mathrm{C} 2$ & & & & $\mathrm{~d}$ & affected by data defect in 1 st spectrum. \\
\hline & & 10274 & $C \mathrm{IV}$ & $\begin{array}{l}\mathrm{r} \\
\mathrm{b}\end{array}$ & C2 & $\begin{array}{l}-2.72 \\
-0.31\end{array}$ & $-2.60 \pm 2.35$ & $-2.60 \pm 2.52$ & a1 & \\
\hline & 2.8245 & 19374 & Civ & $\begin{array}{l}\mathrm{D} \\
\mathrm{r}\end{array}$ & 42 & $\begin{array}{l}-0.31 \\
-1.06\end{array}$ & $\begin{array}{l}-0.27 \pm 0.94 \\
-0.98+2.03\end{array}$ & $\begin{aligned}-0.27 \pm 1.30 \\
0.98+2.29\end{aligned}$ & $\mathrm{~b}$ & margin is $\angle 2 A$ because of line blending. \\
\hline & 2.8283 & 19077 & Si IV & $\mathrm{b}$ & $\mathrm{C} 2$ & -1.37 & $-1.32 \pm 0.52$ & $-1.32 \pm 1.04$ & a1 & \\
\hline & & & & $\mathrm{r}$ & & & & & $\mathrm{d}$ & affected by data defect in 1st spectrum. \\
\hline & 2.8294 & 18991 & C IV & $\mathrm{b}, \mathrm{r}$ & B2 & -0.82 & $-0.83 \pm 0.23$ & $-0.83 \pm 0.93$ & a2 & consider blue and red components together because of line locking. \\
\hline & 2.8347 & 18578 & Si IV & $\mathrm{b}$ & B1 & -1.95 & $-1.92 \pm 0.35$ & $-1.92 \pm 0.97$ & a1 & \\
\hline HE1009+2956 & 10065 & 66707 & 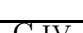 & $\mathrm{r}$ & C1 & -1.85 & $-1.88 \pm 0.53$ & $-1.88 \pm 1.04$ & al & \\
\hline & 1.9005 & (00) & CIV & $\mathrm{r}$ & $\mathrm{Cl}_{1}$ & $\begin{array}{l}-1.11 \\
-0.22\end{array}$ & $\begin{array}{l}-1.13 \pm 0.37 \\
-0.14 \pm 0.59\end{array}$ & $\begin{array}{l}-1.13 \pm 1.06 \\
-0.14 \pm 1.15\end{array}$ & $\begin{array}{l}\text { a1 } \\
\text { a1 }\end{array}$ & \\
\hline & 2.2533 & 33879 & Si IV & $\mathrm{b}$ & $\mathrm{A} 2$ & & & & $\mathrm{~d}$ & blend with other lines. \\
\hline & & & & $\mathrm{r}$ & & -1.71 & $-1.67 \pm 0.40$ & $-1.67 \pm 1.07$ & a1 & \\
\hline & 2.6495 & -452 & $\mathrm{~N} \mathrm{~V}$ & $\begin{array}{l}\mathrm{b} \\
\mathrm{r}\end{array}$ & $\mathrm{A} 2$ & & & & $\begin{array}{l}\mathrm{d} \\
\mathrm{d}\end{array}$ & $\begin{array}{l}\text { affected by data defect in 1st spectrum. } \\
\text { blend with other lines. }\end{array}$ \\
\hline Q1107+4847 & 2.1433 & 70938 & C IV & $\mathrm{b}$ & $\mathrm{C} 2$ & -5.01 & $-5.09 \pm 0.20$ & $-5.09 \pm 1.46$ & a1 & \\
\hline & 2.7243 & 21388 & C IV & $\begin{array}{l}\mathrm{r} \\
\mathrm{b}\end{array}$ & $\mathrm{C} 1$ & & & & $\begin{array}{c}\text { a1 } \\
\text { d }\end{array}$ & affected by data defect in 2 nd spectrum. \\
\hline
\end{tabular}


TABLE 2

\begin{tabular}{|c|c|c|c|c|c|c|c|c|c|c|}
\hline \\
\hline & \multirow[t]{2}{*}{2.7243} & 21388 & Si IV & \multirow{2}{*}{$\begin{array}{l}\mathrm{r} \\
\mathrm{b} \\
\mathrm{r}\end{array}$} & \multirow[t]{2}{*}{$\mathrm{A} 2$} & \multirow[t]{2}{*}{-5.78} & \multirow[t]{2}{*}{$-5.84 \pm 0.58$} & \multirow[t]{2}{*}{$-5.84 \pm 1.56$} & \multirow{2}{*}{$\begin{array}{c}\mathrm{d} \\
\mathrm{a} 1 \\
\mathrm{~d}\end{array}$} & affected by data defect in 2 nd spectrum. \\
\hline & & & & & & & & & & blend with other lines. \\
\hline & 2.7593 & 18595 & Si IV & $\mathrm{b}$ & $\mathrm{C} 1$ & -3.20 & $-3.21 \pm 0.29$ & $-3.21 \pm 1.48$ & $\mathrm{~b}$ & margin is $<2 \AA$ because of line blending. \\
\hline & & & & $\mathrm{r}$ & & -2.61 & $-2.52 \pm 0.39$ & $-2.52 \pm 1.50$ & $\mathrm{~b}$ & margin is $<2 \AA$ because of line blending. \\
\hline & 2.7610 & 18460 & C IV & $\mathrm{b}$ & $\mathrm{C} 1$ & -2.99 & $-2.99 \pm 0.15$ & $-2.99 \pm 1.46$ & a1 & \\
\hline & & & & $\mathrm{r}$ & & -2.24 & $-2.25 \pm 0.13$ & $-2.25 \pm 1.46$ & a1 & \\
\hline & 2.7621 & 18372 & Si IV & $\mathrm{b}$ & $\mathrm{C} 2$ & -3.15 & $-3.16 \pm 0.22$ & $-3.16 \pm 1.47$ & $\mathrm{~b}$ & margin is $<2 \AA$ because of line blending. \\
\hline HS1700+6416 & 2.3154 & 34551 & Si IV & $\frac{\mathrm{r}}{\mathrm{b}}$ & C1 & 0.87 & $0.87 \pm 0.14$ & $0.87 \pm 1.19$ & $\frac{\mathrm{d}}{\mathrm{a} 1}$ & \\
\hline חDI & 2.0104 & 34001 & DIIV & $\begin{array}{l}\mathrm{D} \\
\mathrm{r}\end{array}$ & -1 & 0.25 & $0.25 \pm 0.15$ & $0.25 \pm 1.19$ & a1 & \\
\hline & 2.3156 & 34533 & C IV & $\mathrm{b}$ & $\mathrm{C} 1$ & & & & $\mathrm{~d}$ & affected by data defect in 1 st spectrum. \\
\hline & & & & $\mathrm{r}$ & & -0.40 & $-0.39 \pm 0.16$ & $-0.39 \pm 1.19$ & a1 & \\
\hline & 2.4333 & 24169 & Si IV & $\mathrm{b}$ & $\mathrm{C} 2$ & 0.39 & $0.79 \pm 2.21$ & $0.79 \pm 2.51$ & $\mathrm{c}$ & may be time variable. \\
\hline & 24390 & 23675 & CIV & $\mathrm{r}$ & $\mathrm{B} 2$ & 082 & $0.85+0.26$ & $085+121$ & $\mathrm{~d}$ & blend with other lines. \\
\hline & 2.5785 & 11789 & C IV & $\begin{array}{c}\mathrm{D}, \mathrm{r} \\
\mathrm{b}\end{array}$ & $\begin{array}{l}\mathrm{B} 2 \\
\mathrm{C} 2\end{array}$ & $\begin{array}{l}0.82 \\
1.22\end{array}$ & $\begin{array}{l}0.85 \pm 0.20 \\
1.13 \pm 0.64\end{array}$ & $\begin{array}{l}0.85 \pm 1.21 \\
1.13 \pm 1.34\end{array}$ & $\begin{array}{l}\mathrm{a} 2 \\
\mathrm{a} 1\end{array}$ & \\
\hline & & & & $\mathrm{r}$ & & $\begin{array}{l}1.22 \\
2.16\end{array}$ & $2.08 \pm 1.78$ & $2.08 \pm 2.14$ & $\begin{array}{l}\text { d1 } \\
\text { a1 }\end{array}$ & \\
\hline & 2.7125 & 767 & C IV & $\begin{array}{l}\mathrm{b} \\
\mathrm{r}\end{array}$ & $\mathrm{A} 2$ & 0.31 & $0.32 \pm 0.40$ & $0.32 \pm 1.25$ & $\begin{array}{l}\mathrm{c} \\
\mathrm{d}\end{array}$ & $\begin{array}{l}\text { may be time variable. } \\
\text { affected by data defect in } 2 \text { nd spectrum. }\end{array}$ \\
\hline & 2.7125 & 767 & $\mathrm{~N} \mathrm{~V}$ & $\mathrm{~b}$ & A2 & 2.64 & $2.65 \pm 0.47$ & $2.65 \pm 1.27$ & a1 & \\
\hline & & & & $\mathrm{r}$ & & 1.14 & $1.08 \pm 0.47$ & $1.08 \pm 1.27$ & $\mathrm{a} 1$ & \\
\hline & 2.7164 & 452 & $\mathrm{~N} \mathrm{~V}$ & $\begin{array}{l}\mathrm{b} \\
\mathrm{r}\end{array}$ & A2 & -0.21 & $-0.20 \pm 2.08$ & $-0.20 \pm 2.39$ & $\begin{array}{c}\text { a1 } \\
d\end{array}$ & blend with other lines. \\
\hline
\end{tabular}

\footnotetext{
a Offset velocity that is defined as positive if NALs are blueshifted from the quasar.

Offset velocity that is defined as positive if
Blue (b) or red (r) component of doublet.

c NAL class: Class A, B, or C denote reliable, possible, or intervening NALs, respectively (Misawa et al. 2007a).

d Cross-Correlation Function (Grier et al. 2016).

e Cross-Correlation Centroid Distribution (Grier et al. 2016).

${ }^{\mathrm{f}}$ Velocity shift and its uncertainty after adding an error from spectral distortion. See discussion in $\S$ 的 the text.

$\mathrm{g}$ Sample class: a1) satisfy all criteria, a2) line-locking, b) sampling spectral region is smaller than $2 \AA$ at either or both side of NAL because of other lines or data defect, c) show a hint of time

variability, and d) NAL itself blends other line or data defect.
} 
TABLE 3

Velocity Uncertainty due to Spectrum Distortion

\begin{tabular}{ccc}
\hline \hline Quasar & Number of Pairs $^{a}$ & $\begin{array}{c}\sigma(\Delta v)_{\mathrm{rms}} \\
\left(\mathrm{km} \mathrm{s}^{-1}\right)\end{array}$ \\
\hline HE0130-4021 & 3 & 1.25 \\
Q0450-1310 & 28 & 1.67 \\
HE0940-1050 & 91 & 0.90 \\
HE1009+2956 & 1 & 0.99 \\
Q1107+4847 & 21 & 1.45 \\
HS1700+6416 & 15 & 1.18 \\
& \\
\hline a Roor-mean-square of $|\Delta v|$ between all pairs of \\
class-C lines.
\end{tabular}

TABLE 4

ShIFT VelOCITY OF NALS

\begin{tabular}{|c|c|c|c|c|c|c|c|c|}
\hline \multirow[b]{2}{*}{ Quasar } & \multicolumn{3}{|c|}{ all NALs } & \multicolumn{5}{|c|}{ reliable NALs } \\
\hline & $\begin{array}{c}\Delta v_{\mathrm{AB}^{a}} \\
\left(\mathrm{~km} \mathrm{~s}^{-1}\right)\end{array}$ & $\begin{array}{c}\Delta v_{\mathrm{C}}^{b} \\
\left(\mathrm{~km} \mathrm{~s}^{-1}\right)\end{array}$ & s.1. ${ }^{c}$ & $\begin{array}{c}\Delta v_{\mathrm{AB}}{ }^{a} \\
\left(\mathrm{~km} \mathrm{~s}^{-1}\right)\end{array}$ & $\begin{array}{c}\Delta v_{\mathrm{C}}^{b} \\
\left(\mathrm{~km} \mathrm{~s}^{-1}\right)\end{array}$ & s.l. ${ }^{c}$ & $\begin{array}{c}\Delta v_{\mathrm{AB}}-\Delta v_{\mathrm{C}} \\
\left(\mathrm{km} \mathrm{s}^{-1}\right)\end{array}$ & $\begin{array}{c}\left(\Delta v_{\mathrm{AB}}-\Delta v_{\mathrm{C}}\right) / \Delta t_{\text {rest }} \\
\left(\mathrm{cm} \mathrm{s}^{-2}\right)\end{array}$ \\
\hline $\begin{array}{c}\text { HE0130-4021 } \\
\text { Q0450-1310 } \\
\text { HE0940-1050 } \\
\text { HE1009+2956 } \\
\text { Q1107+4847 } \\
\text { HS1700+6416 }\end{array}$ & $\begin{array}{c}0.45 \pm 0.32 \\
-3.30 \pm 0.38 \\
-1.51 \pm 0.37 \\
-1.67 \pm 1.07^{d} \\
-5.84 \pm 1.56^{d} \\
1.12 \pm 0.45\end{array}$ & $\begin{array}{c}1.28 \pm 0.50 \\
-3.87 \pm 0.30 \\
-0.94 \pm 0.12 \\
-0.68 \pm 0.49 \\
-3.38 \pm 0.39 \\
0.56 \pm 0.31\end{array}$ & $\begin{array}{l}1.40 \sigma \\
1.18 \sigma \\
1.47 \sigma \\
0.84 \sigma \\
1.53 \sigma \\
1.02 \sigma\end{array}$ & $\begin{array}{c}0.79 \pm 0.54 \\
-3.30 \pm 0.38 \\
-1.90 \pm 0.71 \\
-1.67 \pm 1.07^{d} \\
-5.84 \pm 1.56^{d} \\
1.61 \pm 0.71\end{array}$ & $\begin{array}{c}1.33 \pm 0.87 \\
-3.70 \pm 0.38 \\
-0.98 \pm 0.13 \\
-0.68 \pm 0.49 \\
-3.68 \pm 0.65 \\
0.55 \pm 0.35\end{array}$ & $\begin{array}{l}0.53 \sigma \\
0.74 \sigma \\
1.27 \sigma \\
0.84 \sigma \\
1.28 \sigma \\
1.34 \sigma\end{array}$ & $\begin{array}{l}-0.54 \pm 1.02 \\
+0.40 \pm 0.54 \\
-0.92 \pm 0.72 \\
-0.99 \pm 1.18 \\
-2.16 \pm 1.69 \\
+1.06 \pm 0.79\end{array}$ & $\begin{array}{l}-0.0006 \pm 0.0011 \\
+0.0002 \pm 0.0003 \\
-0.0007 \pm 0.0006 \\
-0.0006 \pm 0.0007 \\
-0.0013 \pm 0.0010 \\
+0.0012 \pm 0.0009\end{array}$ \\
\hline
\end{tabular}



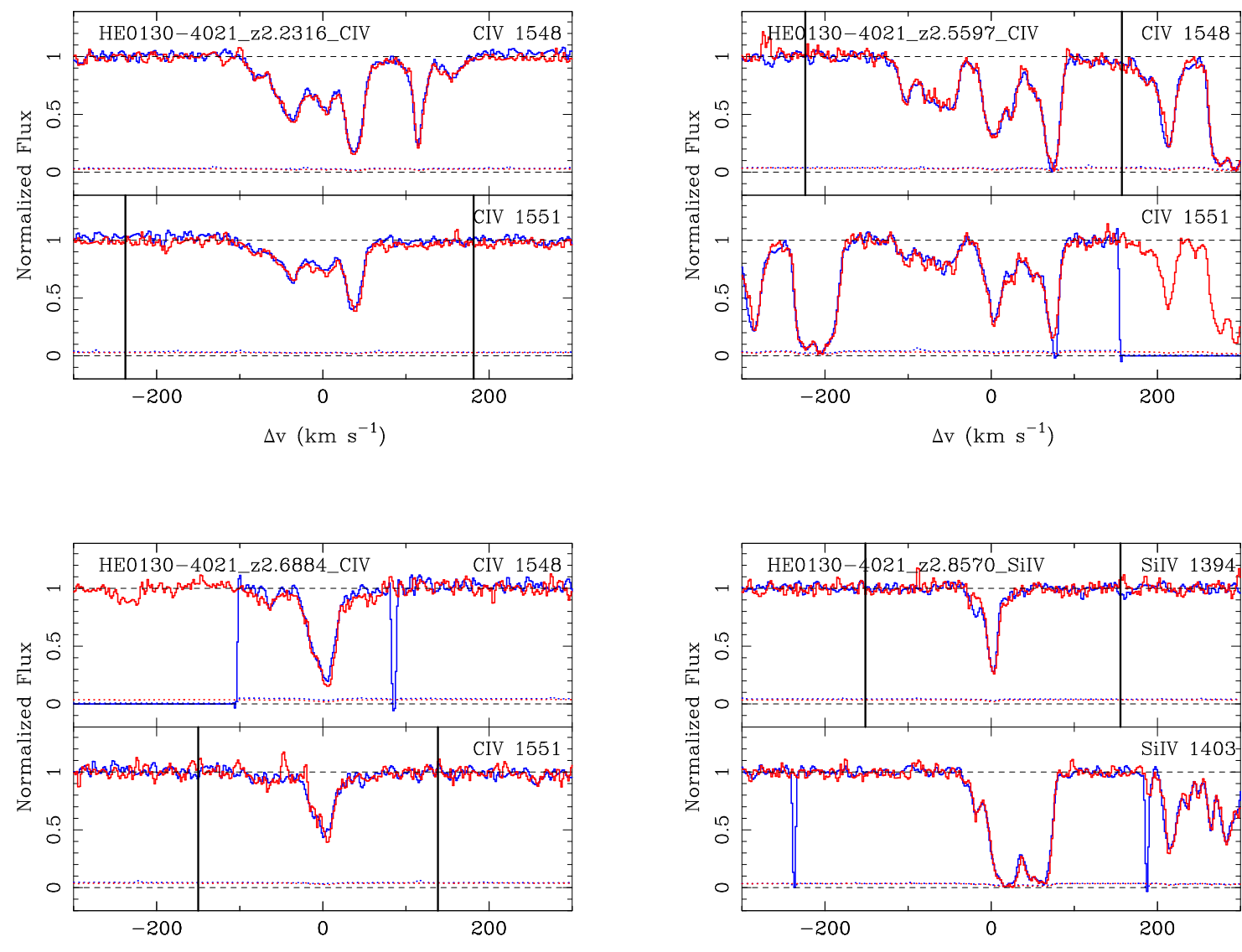

$\Delta \mathrm{v}\left(\mathrm{km} \mathrm{s}^{-1}\right)$

$\Delta \mathrm{v}\left(\mathrm{km} \mathrm{s}^{-1}\right)$
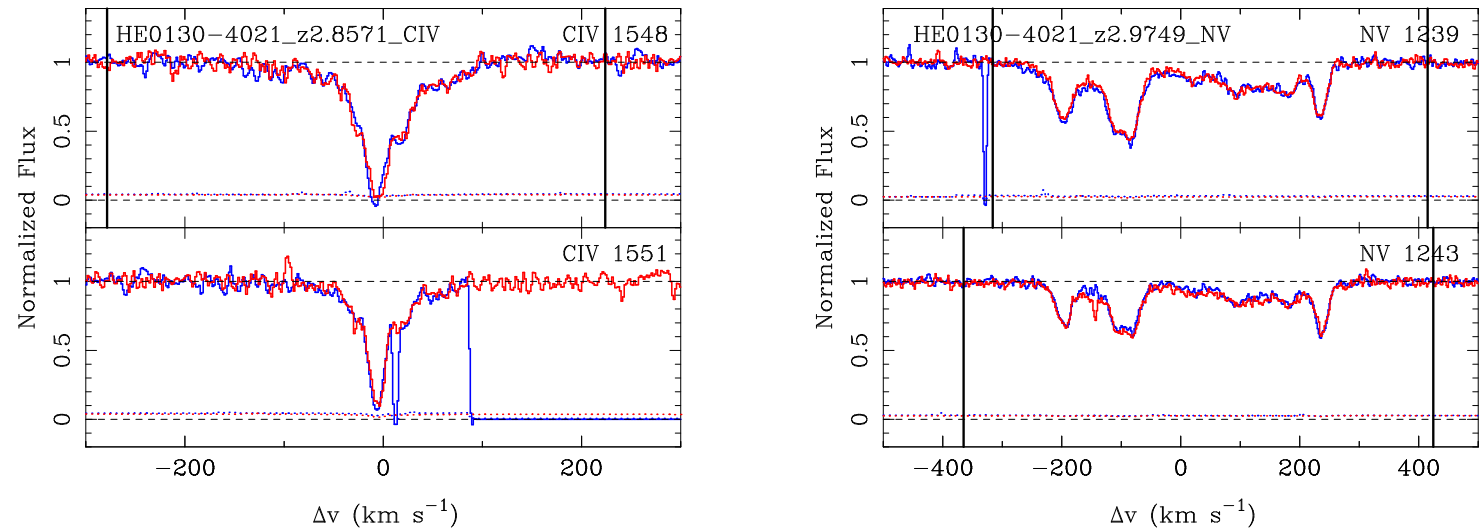

FIG. 1.- Velocity plots of C IV, Si IV, and N V NALs that are included in this work. Blue and red histograms denote the observed spectra in the first and last epochs, respectively. Vertical lines mark the wavelength window over which we performed CCF and CCCD analyses. 

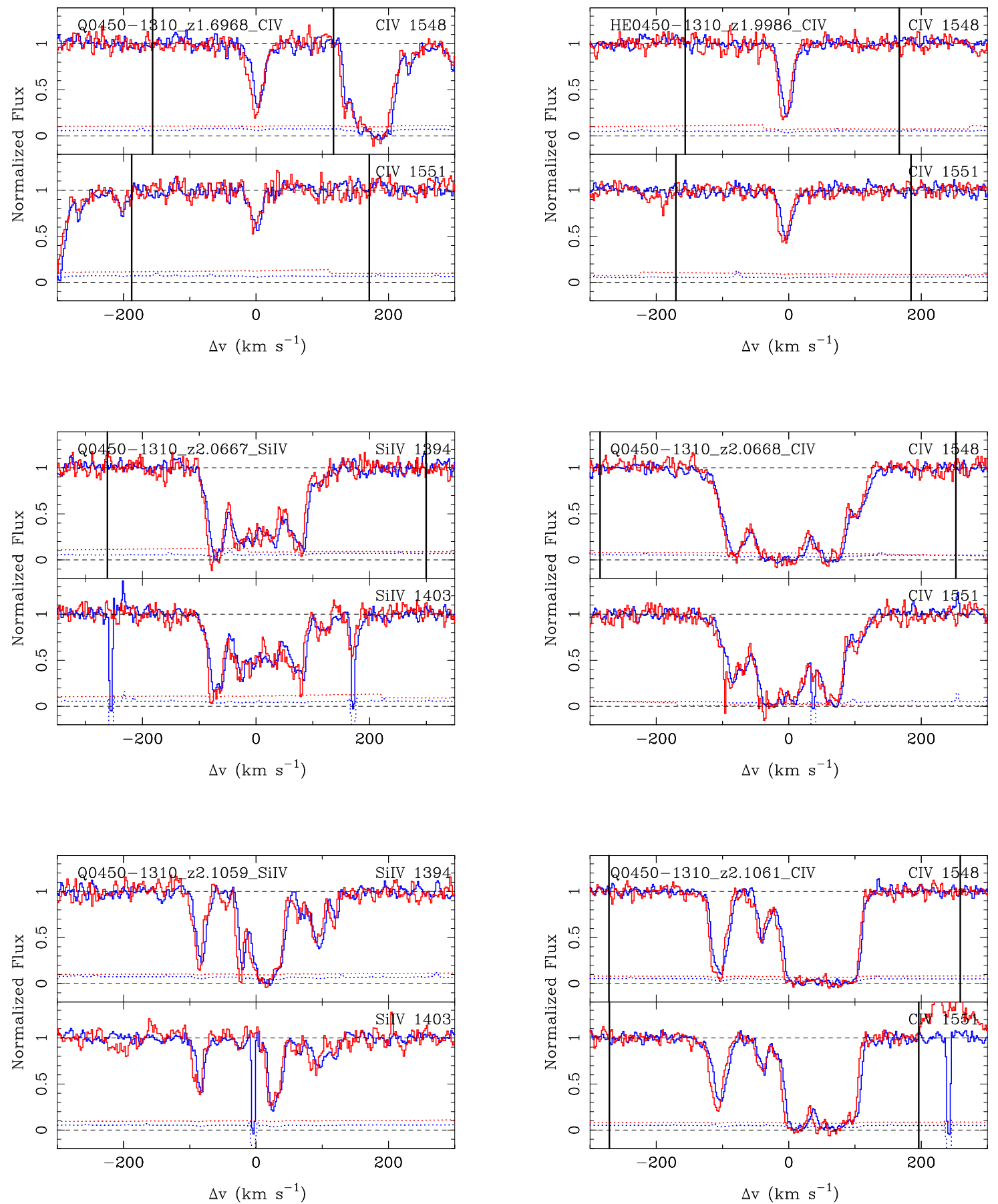

FIG. 1.- continued. 

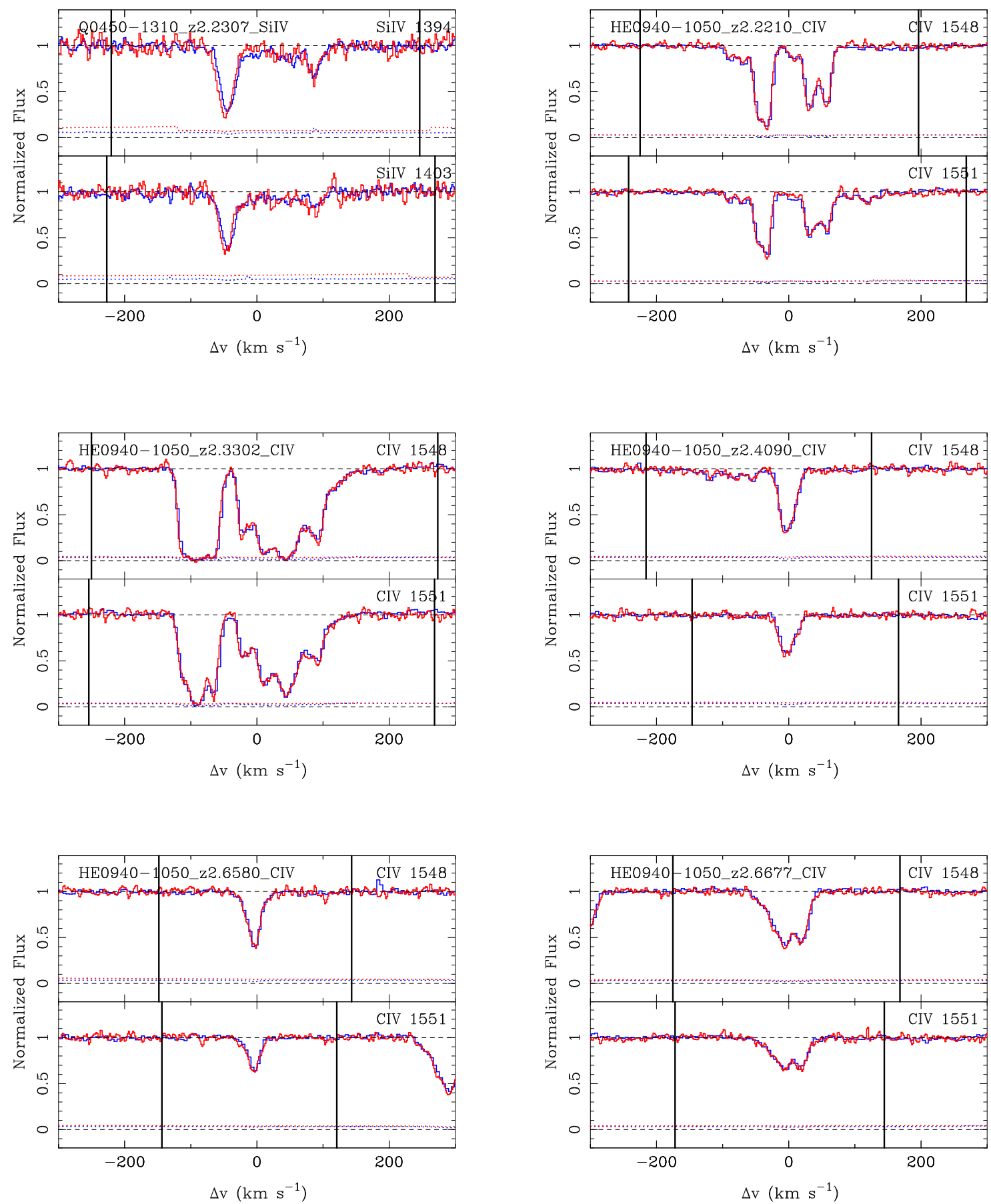

FIG. 1.- continued. 

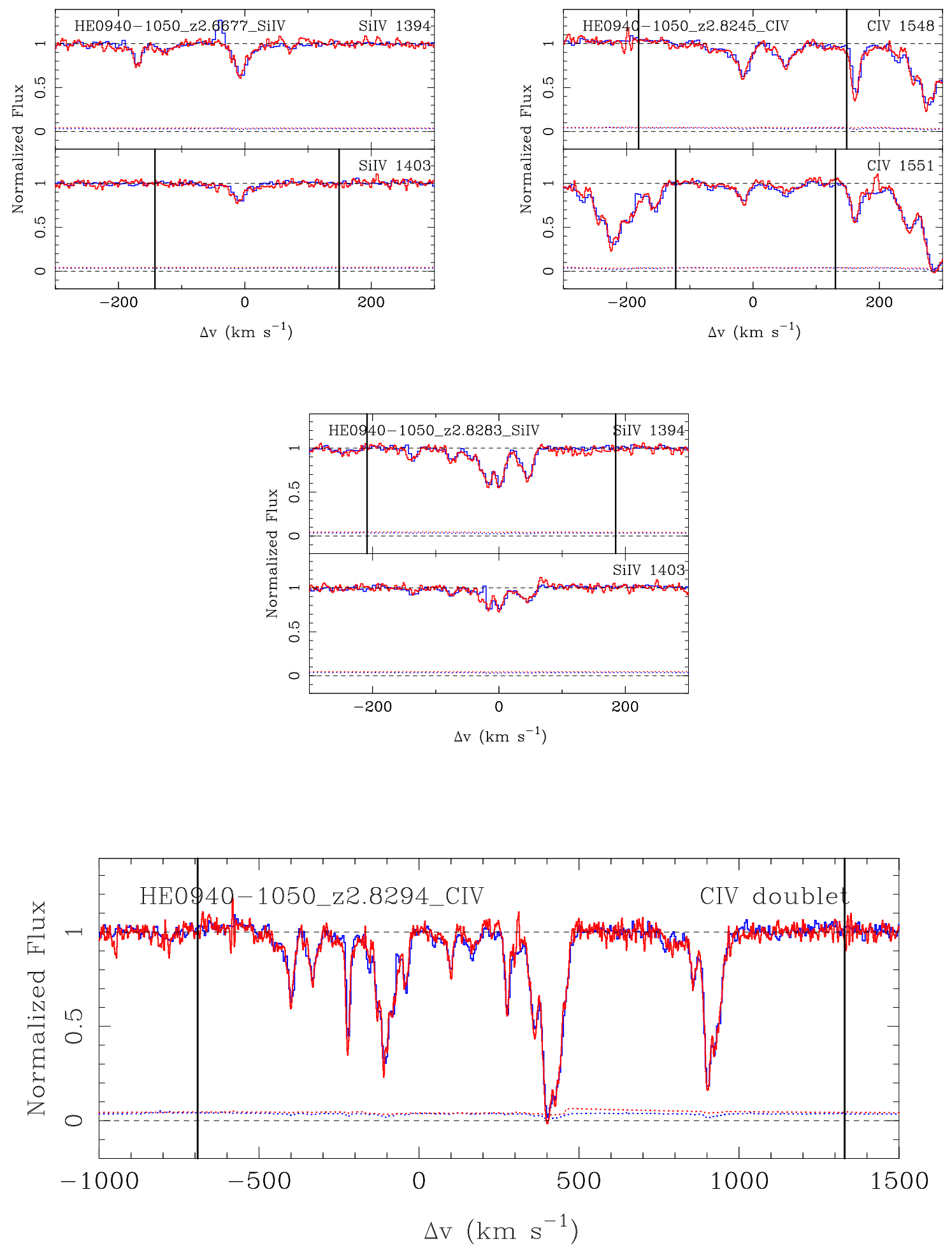

FIG. 1.- continued. 

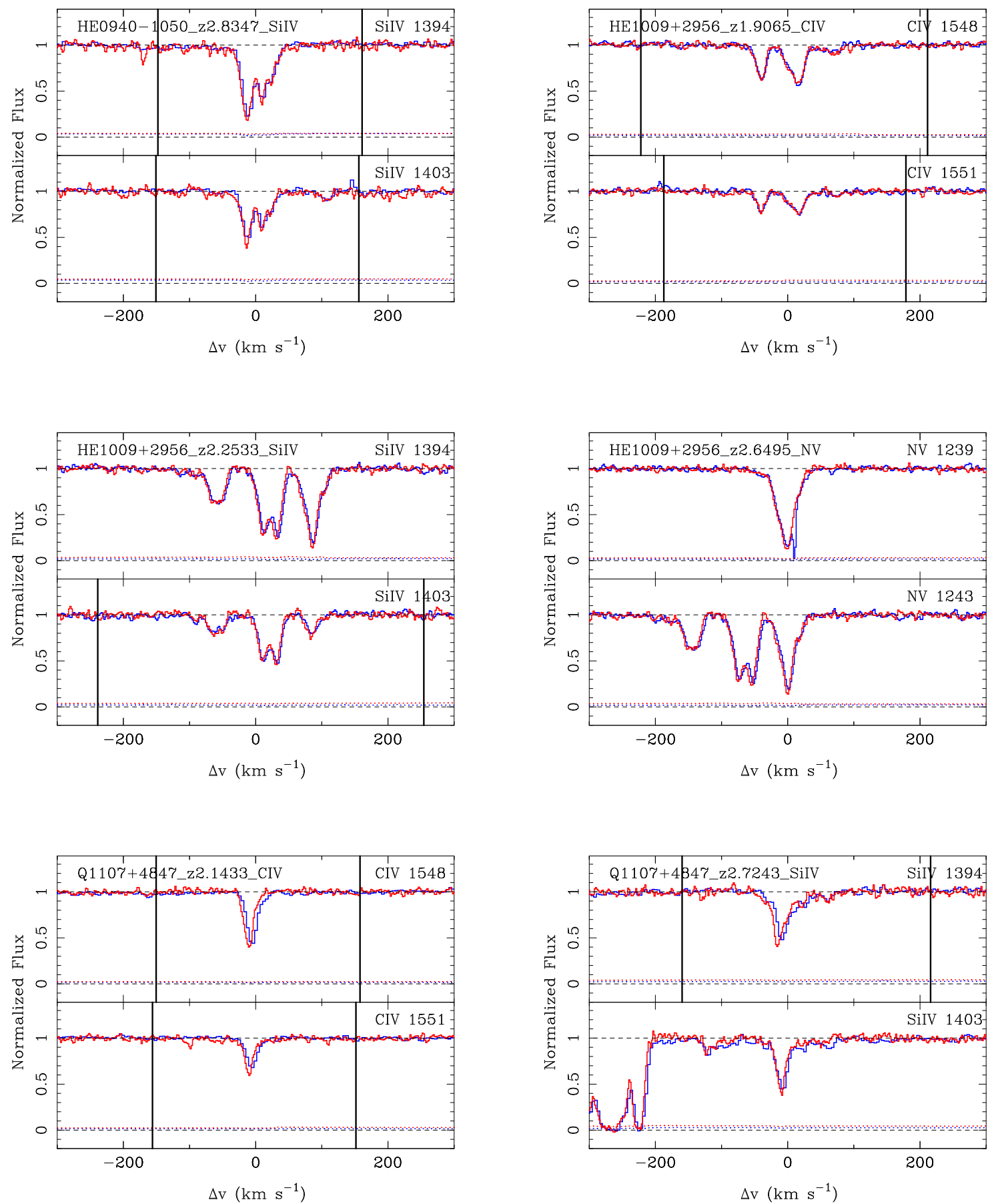

FIG. 1.- continued. 

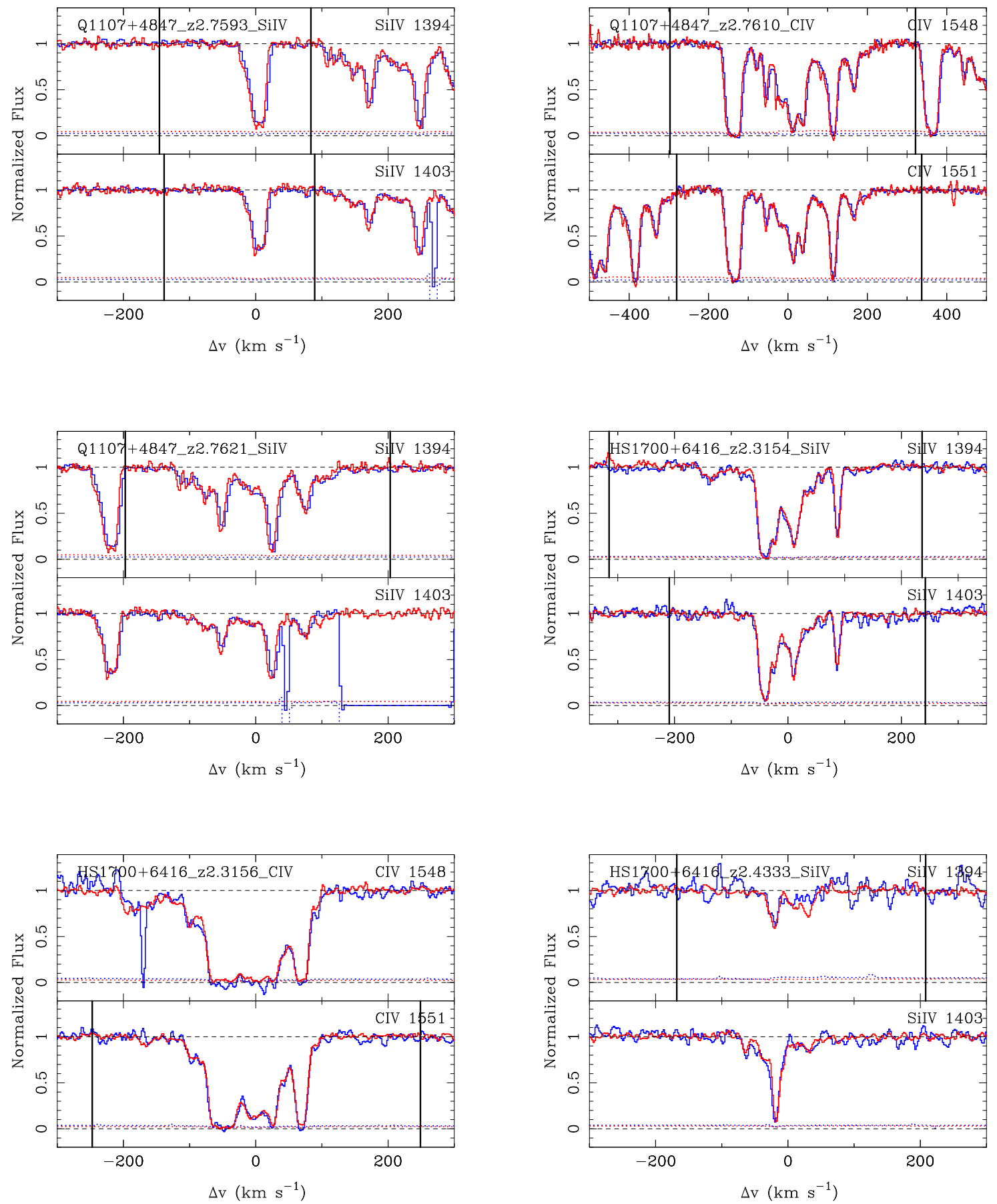

FIG. 1.- continued. 

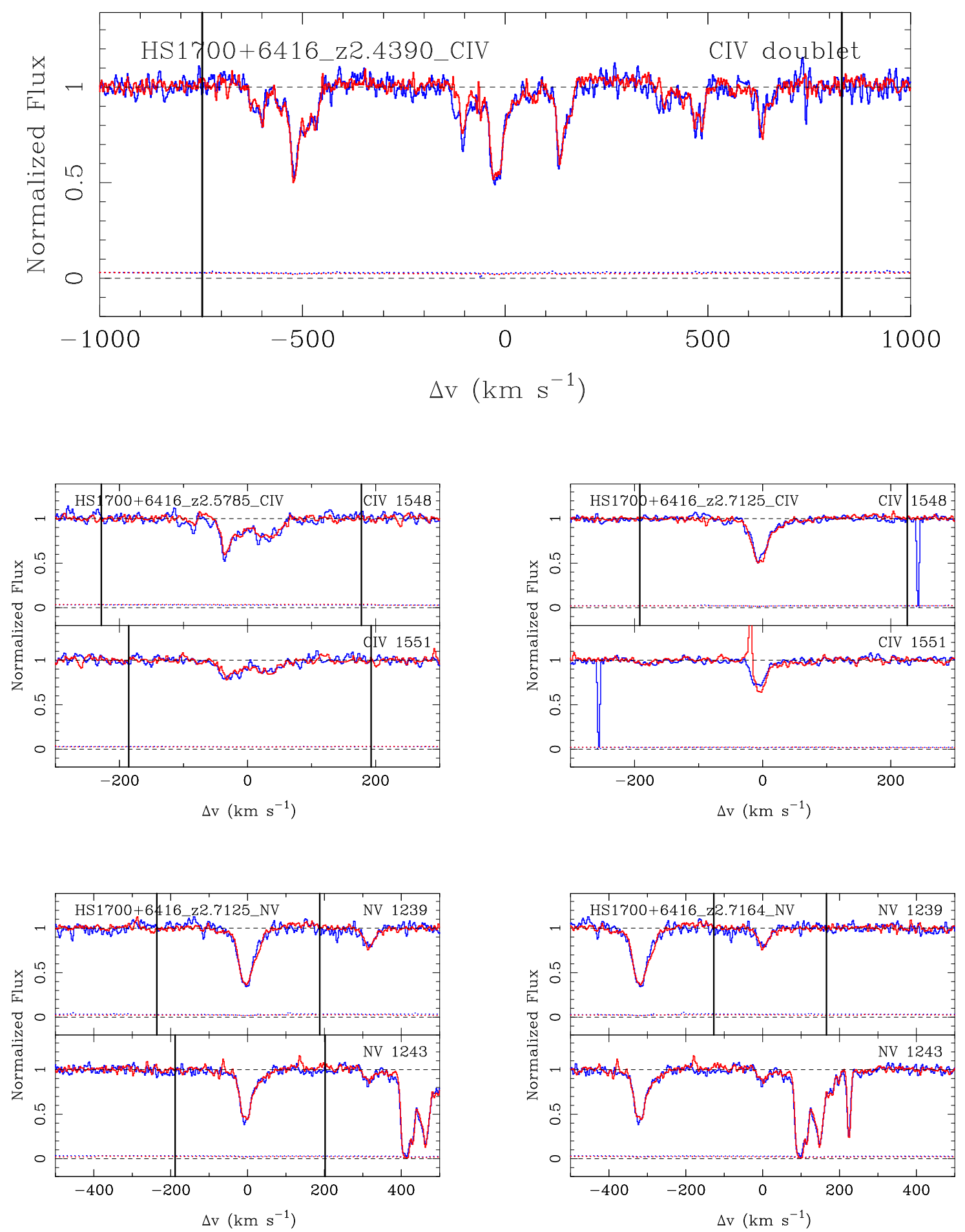

FIG. 1.- continued. 

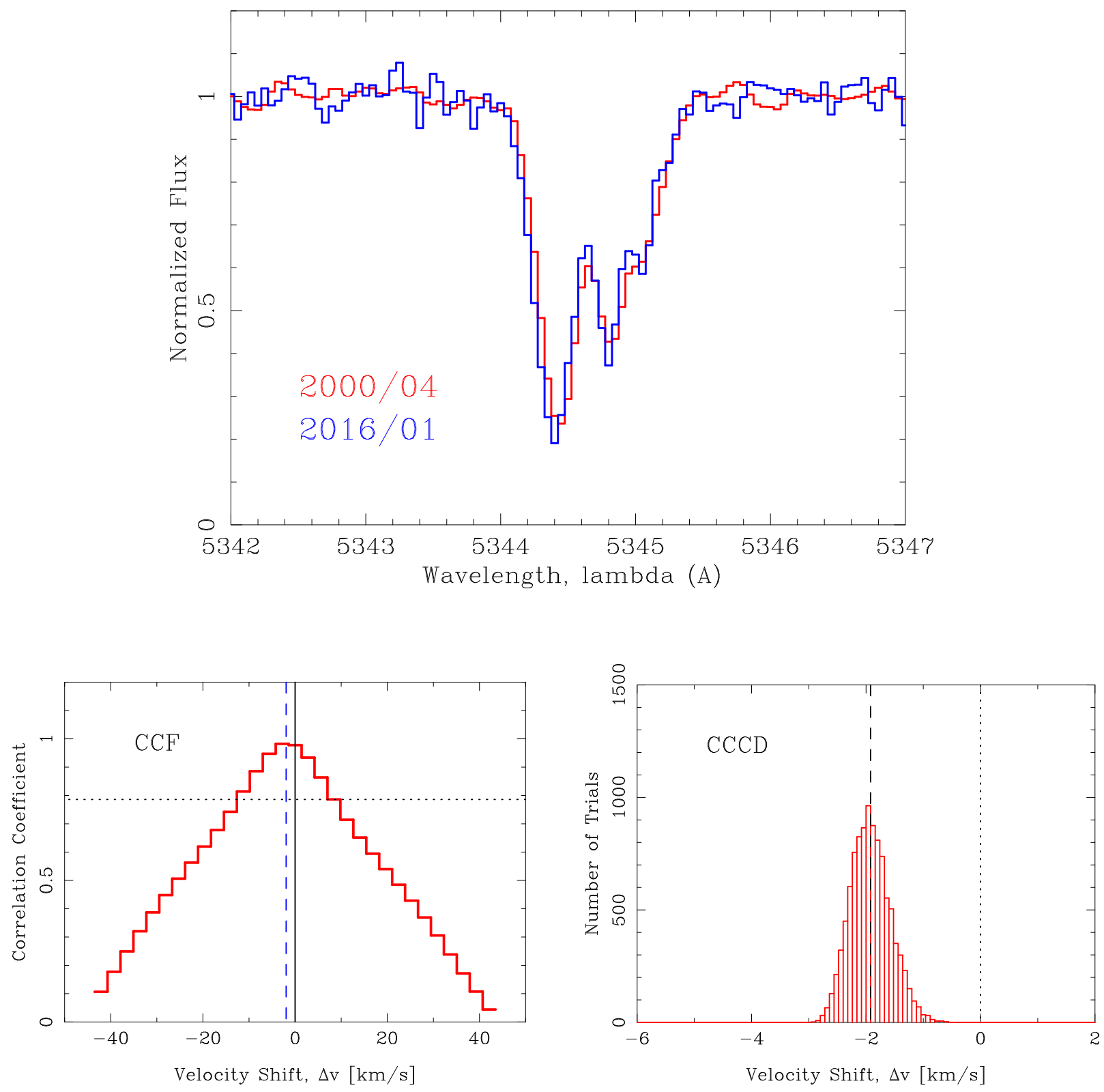

FIG. 2. - The top panel shows absorption profile of the blue-component of the class-B Si IV NAL (i.e., Si IV $\lambda 1394)$ at $z_{\text {abs }}=2.8347$ in HE0940-1050. The spectra were taken on 2000 April 3 and 2016 January 27. The Cross-Correlation Function (CCF) and Cross-Correlation Centroid Distribution (CCCD) for the Si IV $\lambda 1394$ line are shown in the bottom left and right panels, respectively, as an example. 


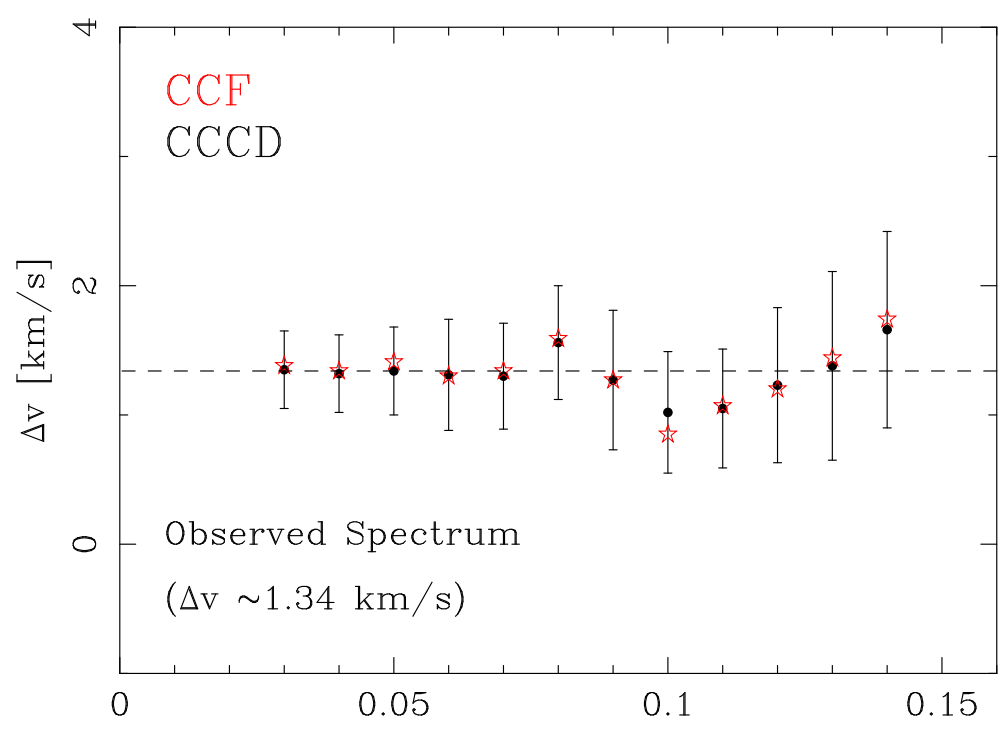

$\Delta \lambda[\AA / \mathrm{bin}]$

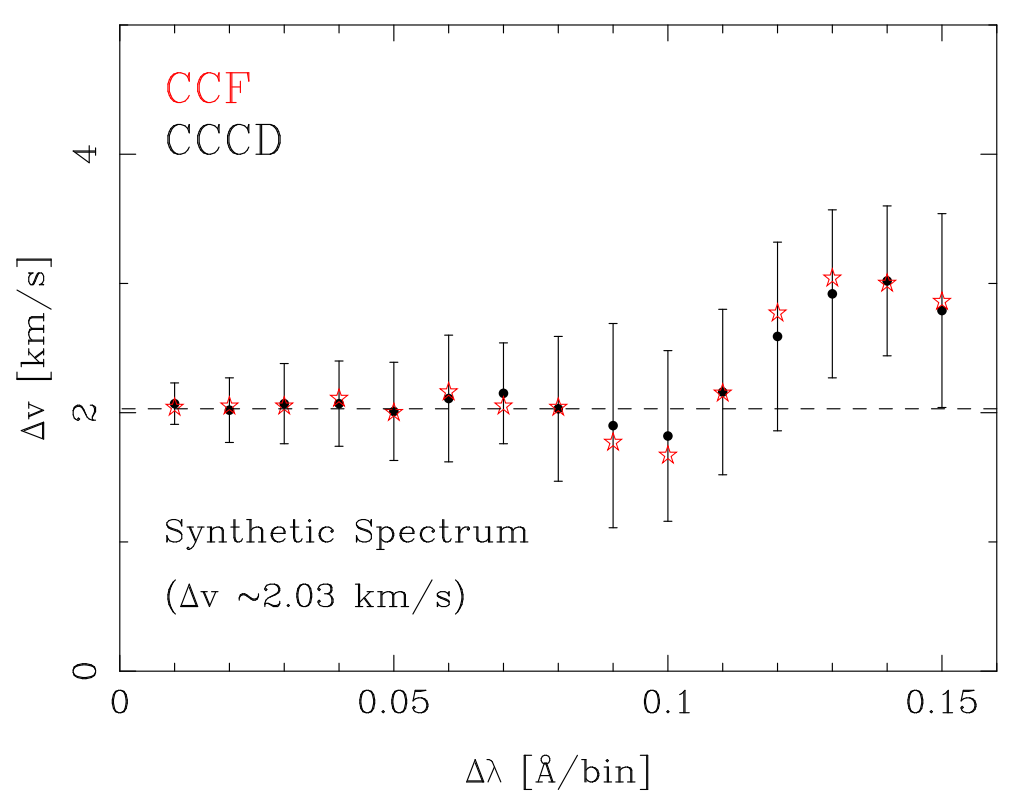

FIG. 3.- Results of tests of the CCF method used in our analysis. The two panels show the measured velocity shift between the two spectra in a pair as a function of dispersion (i.e., size of wavelength bins). An example of results from an observed spectrum, the C IV $\lambda 1548$ line at $z_{\text {abs }}=2.2316$ toward HE0130-4021, is shown in the top panel. An example based on a synthetic spectrum of a similar C IV line with a $2.03 \mathrm{~km} \mathrm{~s}^{-1}$ shift is shown in the bottom panel. See details in $\S$ of the text. 

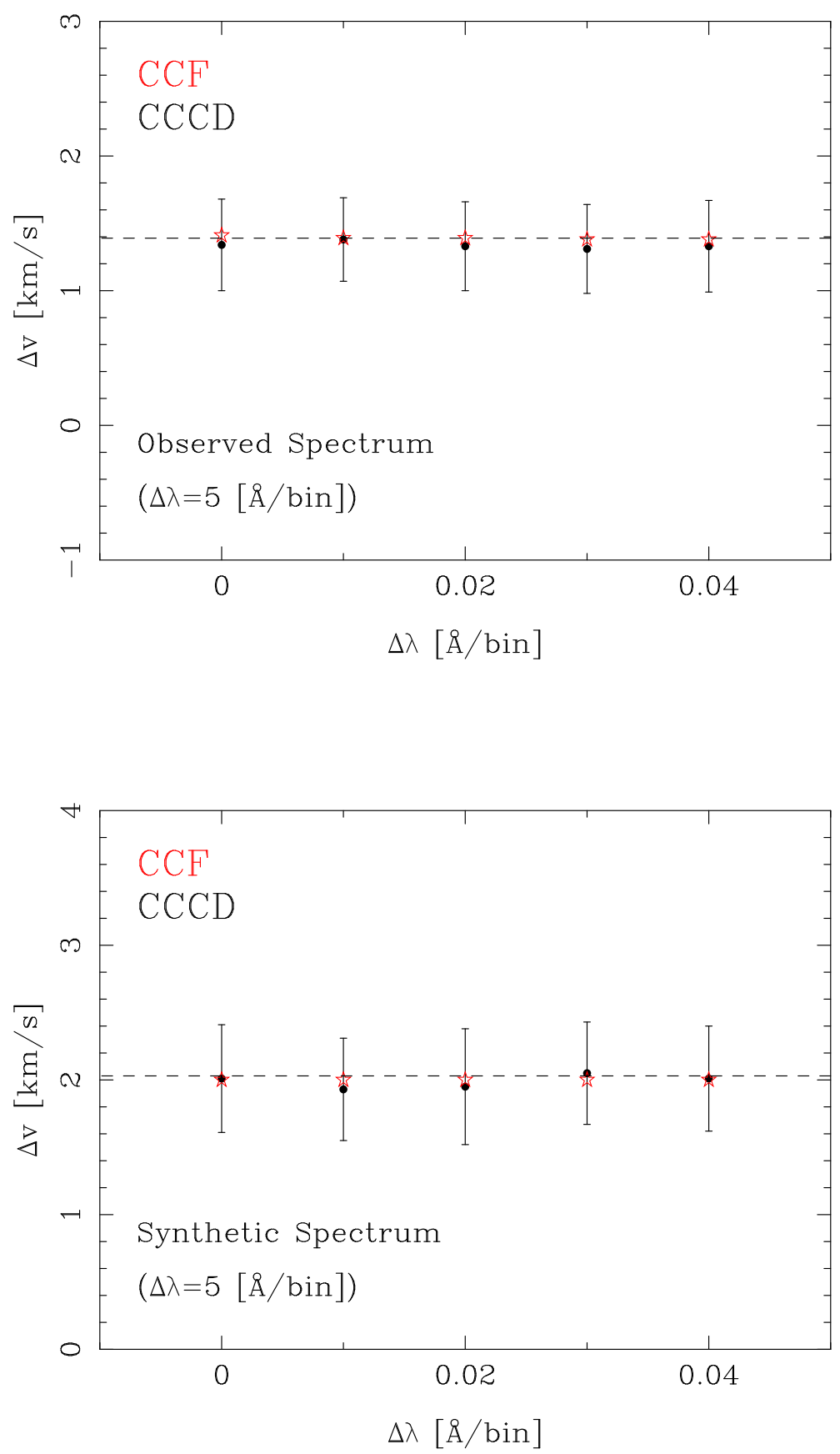

FIG. 4.- Results of tests of the CCF method used in our analysis. The two panels show the measured velocity shift between the two spectra in a pair as a function of starting wavelength. An example of results from an observed spectrum, the C IV $\lambda 1548$ line at $z_{\text {abs }}$ $=2.2316$ toward HE0130-4021, is shown in the top panel. An example based on a synthetic spectrum of a similar C IV line with a $2.03 \mathrm{~km} \mathrm{~s}^{-1}$ shift in the bottom panel. See details in $\S$ of the text. 


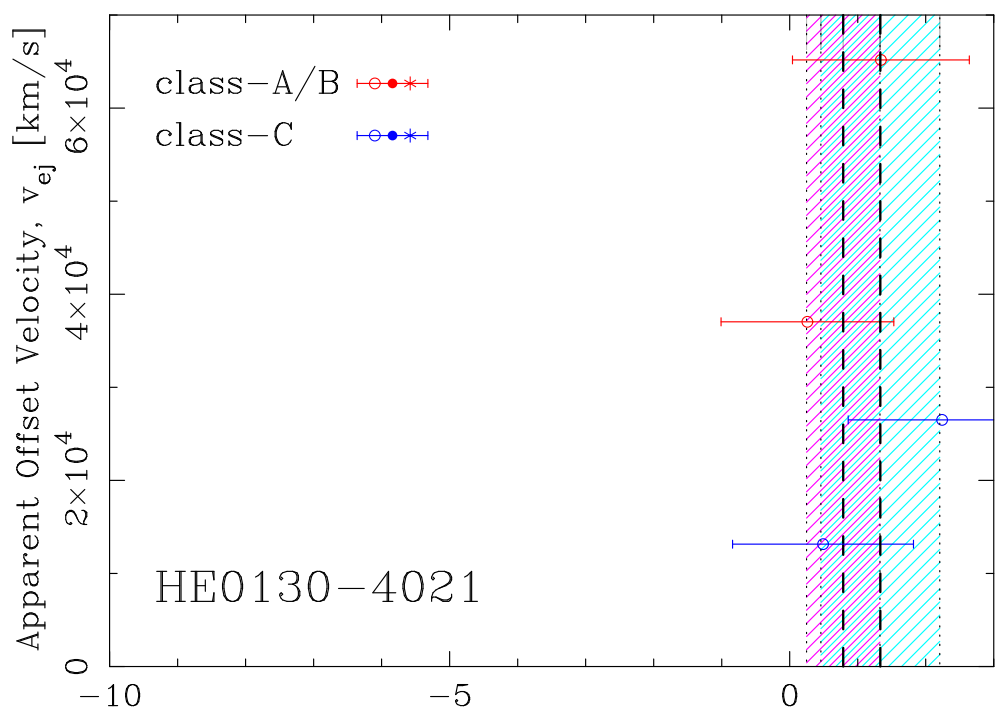

Velocity Shift, $\Delta \mathrm{v}[\mathrm{km} / \mathrm{s}]$

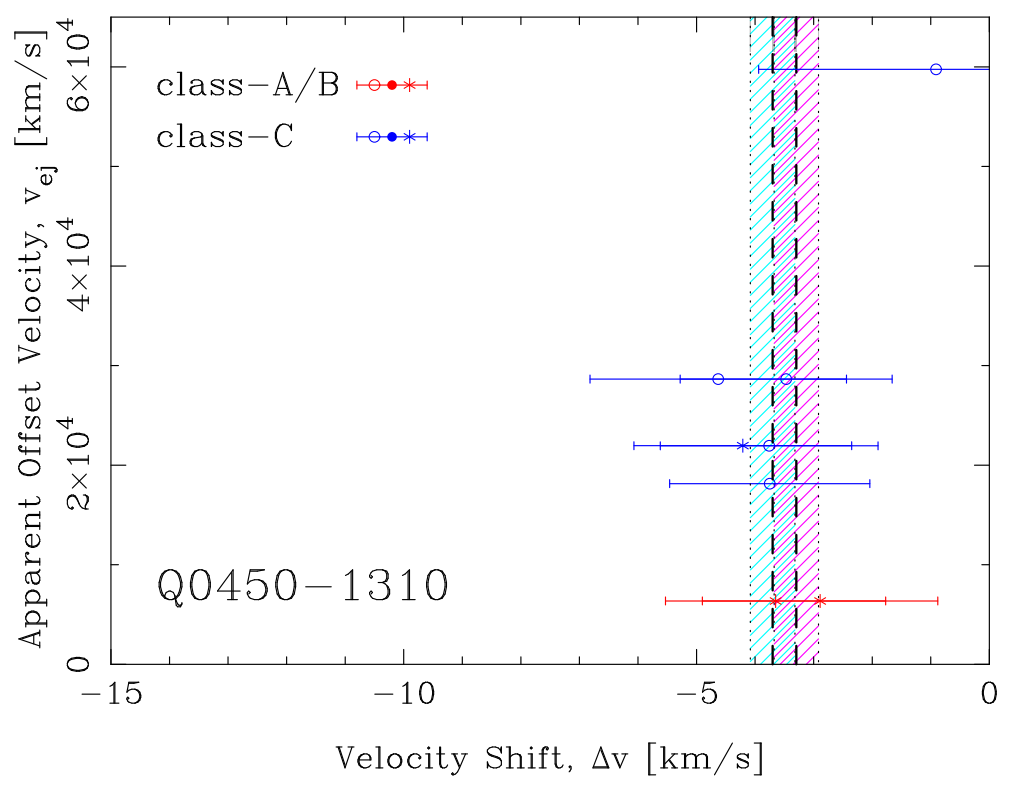

Fig. 5.- Cross-Correlation Centroid Distribution for reliable NALs (points with horizontal $1 \sigma$ errors) in the six quasars of our sample. Open circles, filled circles, and asterisks represent C IV, N V, and Si IV lines, respectively. The horizontal axis denotes velocity shifts of absorption lines between observations (the second epoch relative to the first epoch), while the vertical axis denotes offset velocities from the quasars. If both lines of a doublet are examined or if NALs from multiple transitions in a single absorption system are examined, multiple points are shown along the vertical axis at a same offset velocity. Hashed regions in Magenta and Cyan show the mean $1 \sigma$ errors of velocity shift for class-A/B and class-C NALs between observations that result from the combination of statistical and systematic errors (see Table 4). The center and $\pm 1 \sigma$ boundaries of velocity shifts are shown with vertical dashed and dotted lines. 


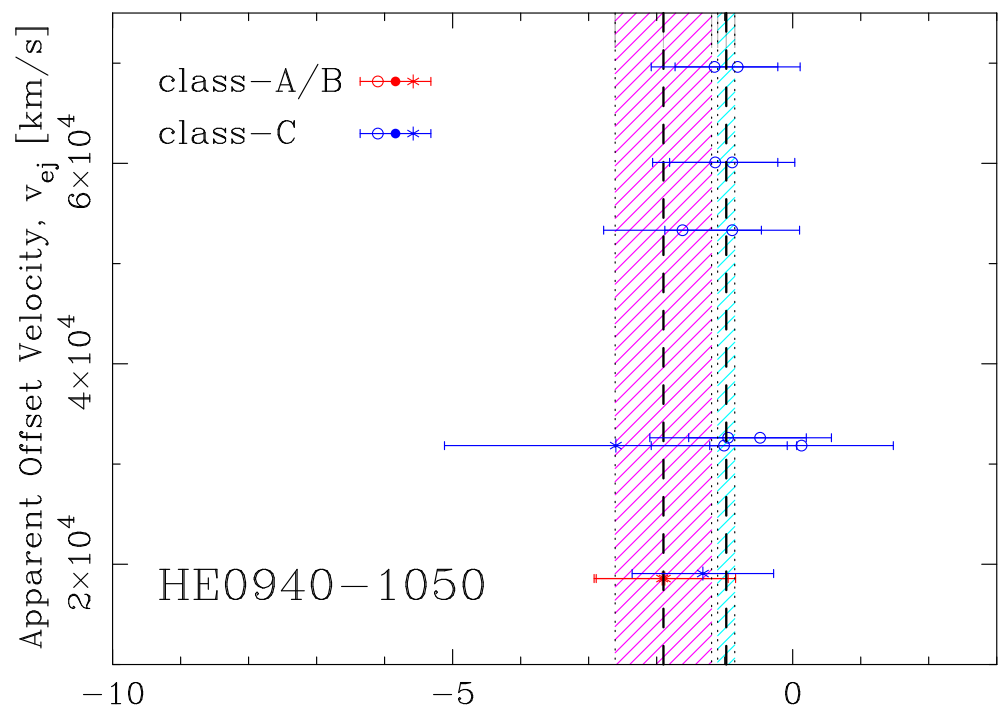

Velocity Shift, $\Delta \mathrm{v}[\mathrm{km} / \mathrm{s}]$

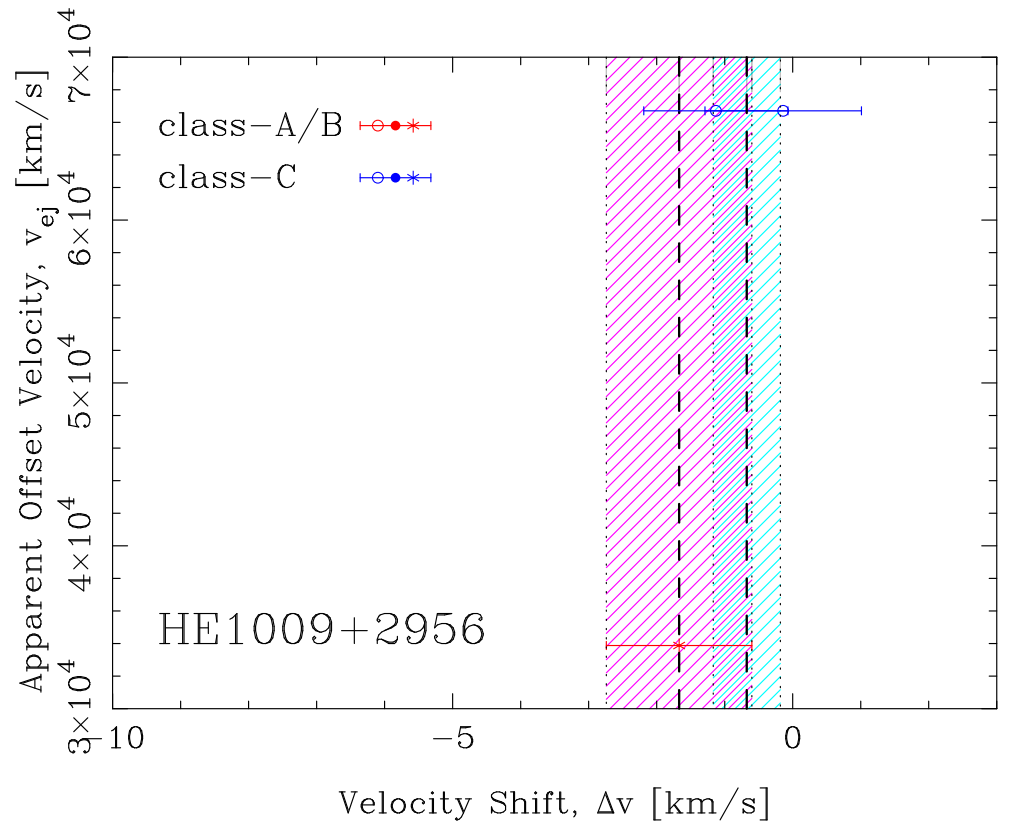

FIG. 5.- continued. 

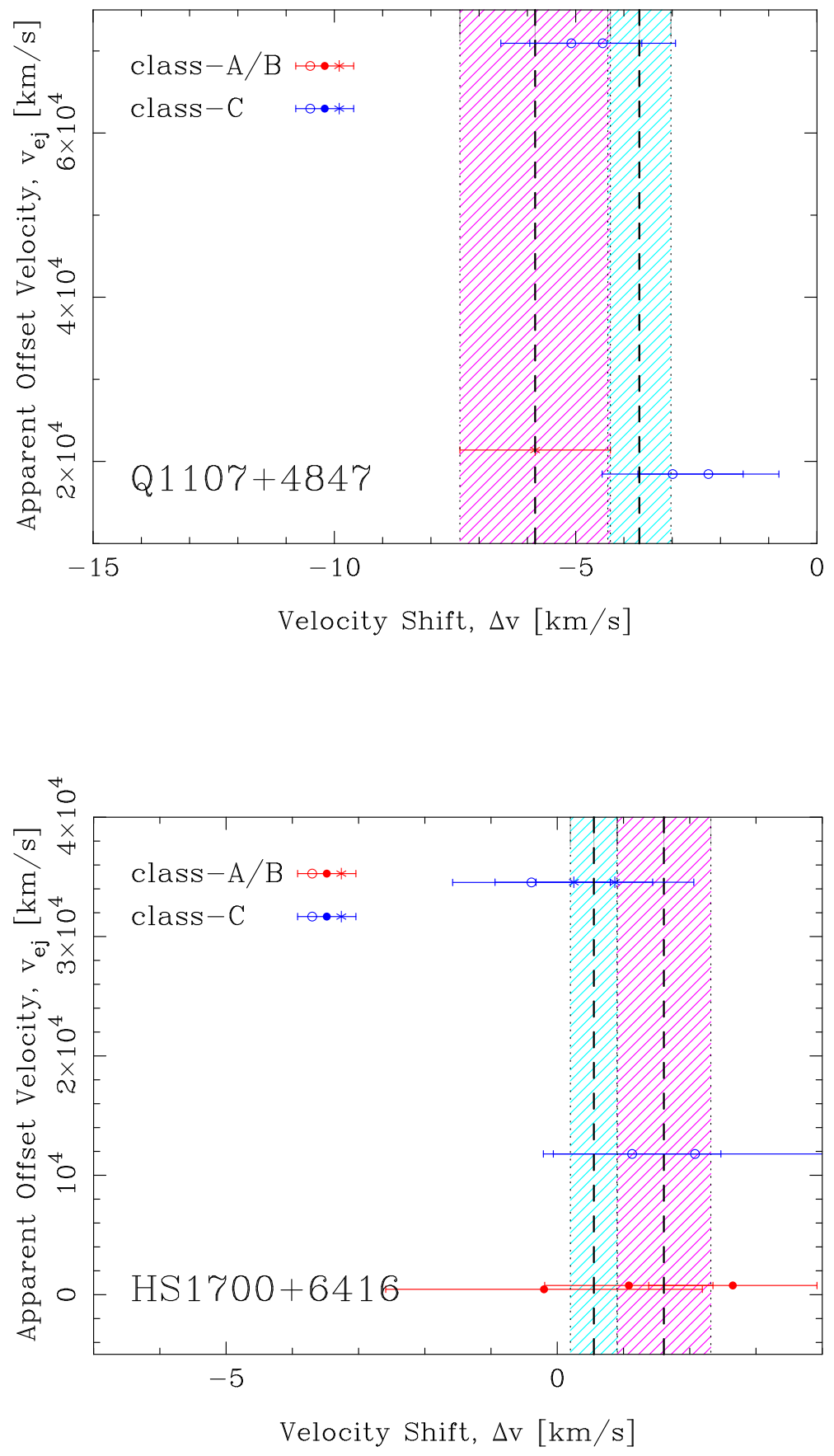

FIG. 5.- continued. 


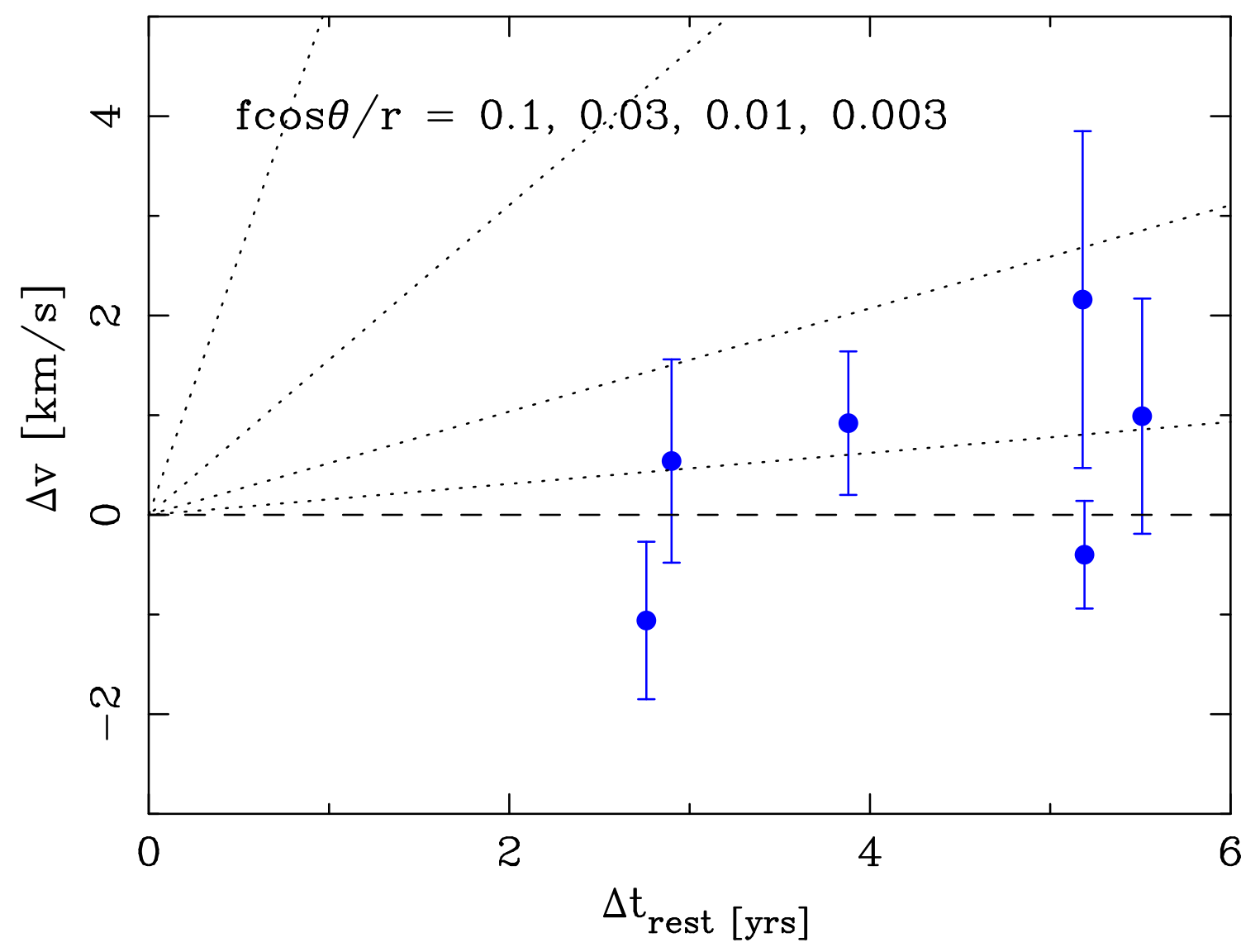

FIG. 6.- Velocity shift of intrinsic NALs between the observations as a function of time interval (filled blue circles). The four dashed lines are the predicted velocity shifts from equation $[7$ with $f \cos \theta / r=0.1,0.03,0.01$, or 0.003 (from top to bottom), for the typical luminosity and black hole mass of our target quasars $\left(L=10^{48} \mathrm{erg} \mathrm{s}^{-1}, M=10^{9} M_{\odot}\right)$ as well as a typical hydrogen column density of NAL absorbers $\left(N_{H}=10^{18} \mathrm{~cm}^{-2}\right)$. 